\title{
Killing superalgebras for lorentzian five-manifolds
}

\author{
Andrew Beckett and José Figueroa-O'Farrill \\ Maxwell Institute and School of Mathematics, The University of Edinburgh, \\ Edinburgh EH9 3FD, Scotland, U.K. \\ E-mail: andy.beckett123@gmail.com, j.m.figueroa@ed.ac.uk
}

ABStract: We calculate the relevant Spencer cohomology of the minimal Poincaré superalgebra in 5 spacetime dimensions and use it to define Killing spinors via a connection on the spinor bundle of a 5-dimensional lorentzian spin manifold. We give a definition of bosonic backgrounds in terms of this data. By imposing constraints on the curvature of the spinor connection, we recover the field equations of minimal (ungauged) 5-dimensional supergravity, but also find a set of field equations for an $\mathfrak{s p}(1)$-valued one-form which we interpret as the bosonic data of a class of rigid supersymmetric theories on curved backgrounds. We define the Killing superalgebra of bosonic backgrounds and show that their existence is implied by the field equations. The maximally supersymmetric backgrounds are characterised and their Killing superalgebras are explicitly described as filtered deformations of the Poincaré superalgebra.

KEywords: Space-Time Symmetries, Differential and Algebraic Geometry, Supergravity Models

ArXiv EPrint: 2105.05775 


\section{Contents}

1 Introduction 1

2 The Poincaré superalgebra $\quad 3$

2.1 Spinorial conventions 3

2.2 The Poincaré superalgebra 9

$\begin{array}{llr}3 & \text { Spencer cohomology } & 9\end{array}$

$\begin{array}{llr}3.1 & \text { Lie algebra cohomology } & 9\end{array}$

$\begin{array}{ll}3.2 & \text { The Spencer complex } \\ \end{array}$

$\begin{array}{ll}3.3 & \text { Solution of the first cocycle condition } \\ \end{array}$

$\begin{array}{ll}3.4 & \text { Solution of the second cocycle condition } \\ \end{array}$

4 Zero-curvature equations 15

$\begin{array}{lll}\text { 4.1 Setup } & 15\end{array}$

$\begin{array}{lll}4.2 & \text { Conventions on curvature tensors } & 18\end{array}$

$\begin{array}{lll}4.3 & \text { Determination of the superconnection curvature } & 19\end{array}$

4.4 Clifford trace of superconnection curvature 22

4.5 Flatness of the superconnection and maximal supersymmetry 25

5 The Killing superalgebra $\quad 26$

$\begin{array}{ll}5.1 & \text { The spinorial Lie derivative } \\ 5.2\end{array}$

$\begin{array}{ll}5.2 & \text { Spinor field bilinears } \\ 5.3 & 27\end{array}$

$\begin{array}{lll}5.3 & \text { Properties of Killing spinors } & 28\end{array}$

$\begin{array}{ll}5.4 & \text { Existence of Killing superalgebras }\end{array}$

6 Maximally supersymmetric backgrounds $\quad 32$

6.1 Maximally supersymmetric supergravity backgrounds 32

6.2 Maximally supersymmetric backgrounds with $F \neq 0$

$\begin{array}{lll}6.2 .1 & \text { Spacelike } \varphi & 33\end{array}$

6.2.2 Timelike $\varphi \quad 34$

$\begin{array}{lll}6.2 .3 \quad \text { Null } \varphi & 34\end{array}$

6.3 Killing superalgebras 35

6.3.1 Killing superalgebras for maximally supersymmetric supergravity backgrounds 36

6.3.2 Killing superalgebras for maximally supersymmetric backgrounds with $\begin{array}{ll}F \neq 0 & 37\end{array}$

$\begin{array}{ll}\text { A Tensorial identities for 2-forms } & 39\end{array}$ 


\section{Introduction}

The interplay between supersymmetry and geometry has a long and beautiful history, but it is fair to say that we are still trying to understand which geometries can support supersymmetric theories. One reason is that the very notion of "supersymmetric theory" is fluid. If we take the conservative stance that a supersymmetric theory is a field theory invariant under some supersymmetry algebra, then the first question one needs to answer is which are the possible supersymmetry algebras, to be followed by the analysis of their unitary representations. The former problem has not been completely solved, whereas the latter problem is largely unexplored.

There are two main classes of supersymmetric theories, depending on whether or not the supersymmetry is local. The former are the supergravity theories, many of which are related to low-energy limits of superstring theories, whereas the latter are the rigidly supersymmetric theories, which are the subject of much study today due to their rôle in localisation in quantum field theory (see, e.g., [1]). The two kinds of theories are closely related. Indeed, one way to construct rigidly supersymmetric theories, pioneered by Festuccia and Seiberg [2] a decade ago, is to couple a supergravity theory (with an off-shell formulation) to matter and then to freeze the gravitational degrees of freedom (i.e., the fields in the supergravity multiplet) in such a way that some supersymmetry is preserved. This results in a rigidly supersymmetric theory for the matter multiplet. Supergravity theories with an off-shell formulation are rare, however, and hence it is desirable to find alternative means to constructing rigidly supersymmetric field theories.

Whereas in the Festuccia-Seiberg approach it is neither essential nor indeed desirable for the fields in the supergravity multiplet to be on-shell, but only for them to preserve some supersymmetry, in the strict context of supergravity the interesting geometries are the supersymmetric bosonic backgrounds. A bosonic background is a solution of the supergravity field equations where the fermionic fields have been put to zero. Bosonic backgrounds have very rich geometries, being after all examples of (higher-dimensional) general relativity coupled to matter.

A particularly interesting and rich subclass of bosonic backgrounds are those which preserve some supersymmetry. Since fermions are set to zero, the variation of any bosonic field under supersymmetry is automatically zero, but not so for the variation of the fermionic fields. In particular, the characteristic property of a supergravity theory is that the variation of the gravitino $\Psi$ under a supersymmetry transformation with spinor field parameter $\varepsilon$ takes the form $\delta_{\varepsilon} \Psi=\mathscr{D} \varepsilon$, where $\mathscr{D}$ is a connection on spinors which, on a bosonic background, includes terms depending on the additional bosonic fields in the supergravity multiplet. For such a transformation to preserve a bosonic background, this variation must vanish on that background; in other words, $\varepsilon$ must be parallel with respect to $\mathscr{D}$. The condition $\mathscr{D} \varepsilon=0$, possibly augmented by algebraic conditions coming from the supersymmetric variations of any other fermionic fields in the supergravity multiplet, is the Killing spinor equation. The spinors $\varepsilon$ obeying it are called Killing spinors, because squaring such a spinor gives rise to a Killing vector field, known as its Dirac current.

It is always the case, perhaps after imposing some additional conditions on the bosonic fields, that the Dirac current of a Killing spinor preserves the other bosonic fields of the 
background, and hence it preserves the connection $\mathscr{D}$. This implies that such Killing vectors preserve the space of Killing spinors, together with which they generate a Lie superalgebra known as the Killing superalgebra of the background [3]. The Killing superalgebra is a useful algebraic invariant of a supersymmetric supergravity background, and one consequence of the homogeneity theorem [4] is that it determines a $\left(>\frac{1}{2}\right)$-BPS ${ }^{1}$ background up to local isometry. This was proved in [5] for eleven-dimensional supergravity, but it holds in general for any background for which the Killing superalgebra is transitive.

The construction of the Killing superalgebra suggests that all we need in order to identify which geometries can support rigid supersymmetry is a suitable notion of Killing spinor: one which guarantees that the Killing spinors generate a Lie superalgebra. ${ }^{2}$ For example, in the standard Poincaré supersymmetry on Minkowski spacetime, Killing spinors are parallel with respect to the spin connection, whereas in AdS supersymmetry [7] Killing spinors are so-called geometric Killing spinors, satisfying $\nabla_{X} \varepsilon=\lambda X \cdot \varepsilon$, for some constant $\lambda$ related to the curvature of AdS. Geometric Killing spinors were also used in the pioneering work of Blau's [8] for the construction of rigidly supersymmetric gauge theories. Parallel and geometric Killing spinors are intrinsic notions on any spin manifold, but the resulting theories are not too different from Poincaré supersymmetry. To make further progress we need to consider other notions of Killing spinors.

If we assume that the definition of a Killing spinor is that it be parallel with respect to a suitable connection in the spinor bundle (possibly augmented by algebraic - i.e., non-differential - constraints), then a straightforward generalisation of the result in [5] for the Killing superalgebra of eleven-dimensional supergravity backgrounds shows that the resulting superalgebra has a special algebraic structure. Namely, it is naturally filtered in such a way that the associated graded superalgebra is a graded subalgebra of the Poincaré superalgebra. We say that it is a filtered subdeformation of the Poincaré superalgebra.

Such deformations are governed by the (generalised) Spencer cohomology of graded superalgebras $[9,10]$, and so calculation of the relevant Spencer cohomology groups for the Poincaré superalgebra is the first step in this analysis. The Spencer cohomology not only determines the filtered deformations of the Poincaré superalgebra, it also gives the expression for the connection defining the notion of a Killing spinor. In some cases, such as the $D=11[11,12]$ and minimal $D=4[13]$ Poincaré superalgebras, one obtains precisely the connection $\mathscr{D}$ of a supergravity theory, but in other cases, such as the minimal $D=$ 6 [14] Poincaré superalgebra, the Spencer cohomology is richer: additional bosonic fields may be turned on, and the definitions of Killing spinors, supersymmetric backgrounds and Killing superalgebras may be consistently generalised to accommodate them. The existence of such generalisations is intriguing, not least because they provide curved backgrounds for rigidly supersymmetric theories which do not appear to be attainable via supergravity.

In the present paper we discuss the Spencer cohomology of the minimal $D=5$ Poincaré superalgebra. Our motivation is two-fold. On the one hand, it is an intermediate case

\footnotetext{
${ }^{1}$ I.e., any background where the dimension of the space of Killing spinors is more than half the rank of the spinor bundle.

${ }^{2}$ For a different approach to this problem, based on the algebraic classification of supersymmetric extensions of known spacetime algebras, and the superisation of the corresponding homogeneous spacetimes, see, e.g., [6].
} 
between two similar calculations: minimal $D=4$ and $D=6$ Poincaré superalgebras, and provides a useful additional datapoint in framing a conjecture about the behaviour of Spencer cohomology under dimensional reduction. A second motivation is that we may then go on to study supersymmetric reductions of the geometries admitting maximal supersymmetry to four dimensions and perhaps in this way obtain novel four-dimensional lorentzian and riemannian spin manifolds admitting rigid supersymmetry. This is the subject of ongoing work.

We find that the relevant Spencer cohomology group $H^{2,2}\left(\mathfrak{s}_{-}, \mathfrak{s}\right)$ is parametrised by a two-form, which is expected from supergravity, and an $\mathfrak{s p}(1)$-valued one-form, which does not correspond to any supergravity field. This is reminiscent of the Spencer data from minimal $D=6$, which also includes an additional $\mathfrak{s p}(1)$-valued one-form [14]. As in the 6-dimensional case, after using Spencer cocycles to define a connection on spinors, by imposing a constraint (the vanishing of the Clifford trace) on the curvature of that connection, the bosonic equations of motion for supergravity can be recovered along with an additional set of field equations for the one-form.

This paper is organised as follows. In section 2, we introduce our conventions and some identities before describing the minimal Poincaré superalgebra $\mathfrak{s}$ in 5 dimensions as a graded Lie superalgebra. Our calculation of the Spencer cohomology $H^{2,2}\left(\mathfrak{s}_{-}, \mathfrak{s}\right)$ is given in section 3 and culminates in Theorem 9. This data is interpreted geometrically in section 4 by using it to define a connection $\mathscr{D}$ on spinors, as well as an associated notion of Killing spinors, in a suitable geometric setting (a bosonic background). The curvature of $\mathscr{D}$ is explicitly calculated in terms of the bosonic background fields and various conditions on it are characterised. Theorem 13 characterises those geometries where the Clifford trace of the curvature vanishes, whereas Theorem 14 characterises those geometries with vanishing curvature. Section 5 is concerned with Killing superalgebras: the spinorial Lie derivative is defined and some of its properties described; some properties of Killing spinors are derived; then finally Killing superalgebras are defined, and their existence is proven (Theorem 21). In section 6 , we describe the maximally supersymmetric backgrounds explicitly. As can be read in Theorem 23, these fall into two branches: the first coincides with maximally supersymmetric supergravity backgrounds, making contact with known results $[15,16]$; the second is characterised by the existence of an $\mathfrak{s p}(1)$-valued one-form. We determine backgrounds belonging to the second branch, noting the resemblance to the 6-dimensional case [14], and we describe the Killing superalgebras in both branches explicitly as filtered deformations of $\mathfrak{s}$. Appendix A is a compilation of combinatorial tensor identities used in geometric calculations.

\section{The Poincaré superalgebra}

In this section we set up our conventions and introduce the Poincaré superalgebra.

\subsection{Spinorial conventions}

Let $(V, \eta)$ be a five-dimensional (mostly minus) lorentzian vector space. We will let $b$ : $V \rightarrow V^{*}$ denote the musical isomorphism sending $v$ to $v^{b}$, where

$$
v^{b}(w)=\eta(v, w) .
$$


We define $\mathfrak{s o}(V)$ to be the Lie algebra of $\eta$-skew-symmetric endomorphisms of $V$ :

$$
\mathfrak{s o}(V)=\{A: V \rightarrow V \mid \eta(A v, w)=-\eta(v, A w) \quad \forall v, w \in V\}
$$

There is a vector space (in fact, an $\mathfrak{s o}(V)$-module) isomorphism $\mathfrak{s o}(V) \cong \wedge^{2} V$. If $A \in \mathfrak{s o}(V)$, we define $\omega_{A} \in \wedge^{2} V$ by

$$
A v=-\iota_{v^{b}} \omega_{A} .
$$

Conversely, if $\omega \in \wedge^{2} V$, we define $A_{\omega} \in \mathfrak{s o}(V)$ by the same relationship: namely,

$$
A_{\omega} v=-\iota v^{b} \omega .
$$

It then follows that these two maps are mutual inverses: $A_{\omega_{A}}=A$ and $\omega_{A_{\omega}}=\omega$. Relative to an orthonormal basis $\boldsymbol{e}_{\mu}$ for $V$, with $\eta\left(\boldsymbol{e}_{\mu}, \boldsymbol{e}_{\nu}\right)=\eta_{\mu \nu}$, we find that

$$
\omega_{A}=\frac{1}{2} A^{\mu \nu} \boldsymbol{e}_{\mu} \wedge \boldsymbol{e}_{\nu} \quad \text { where } \quad A \boldsymbol{e}_{\mu}=\boldsymbol{e}_{\nu} A_{\mu}^{\nu}
$$

and indices are lowered and raised with $\eta_{\mu \nu}$ and its inverse $\eta^{\mu \nu}$.

We define the Clifford algebra $C \ell(V)$ by the Clifford relations (notice the sign!)

$$
v \cdot v=\eta(v, v) \mathbb{1}
$$

As a real associative algebra, $C \ell(V) \cong \operatorname{End}(\Sigma) \oplus \operatorname{End}\left(\Sigma^{\prime}\right)$, where $\Sigma$ and $\Sigma^{\prime}$ are two inequivalent irreducible Clifford modules, which are two-dimensional quaternionic (right) vector spaces. They are distinguished by the action of the centre of $C \ell(V)$. The centre is spanned by the identity and the volume element, defined by the Levi-Civita symbol $\epsilon_{\mu \nu \rho \sigma \tau}$ normalised to $\epsilon_{01234}=1$.

On the Clifford module $\Sigma$ the volume element acts like the identity endomorphism id ${ }_{\Sigma}$, whereas on $\Sigma^{\prime}$ it acts like - id $\Sigma_{\Sigma^{\prime}}$. In other words, the centre of $C \ell(V)$ acts trivially on $\Sigma$ and non-trivially on $\Sigma^{\prime}$. We will work with $\Sigma$ from now on. We will also use the notation $\mathbb{1}$ for the identity endomorphism of $\Sigma$.

Under the representation homomorphism $C \ell(V) \rightarrow \operatorname{End}(\Sigma)$ the basis element $\boldsymbol{e}_{\mu}$ is represented by the endomorphism $\Gamma_{\mu}$. These endomorphisms satisfy the Clifford relation

$$
\Gamma_{\mu} \Gamma_{\nu}+\Gamma_{\nu} \Gamma_{\mu}=2 \eta_{\mu \nu} \mathbb{1}
$$

In addition, they obey

$$
\Gamma_{\mu} \Gamma_{\nu}=\Gamma_{\mu \nu}+\eta_{\mu \nu} \mathbb{1}
$$

with $\Gamma_{\mu \nu}=\frac{1}{2}\left[\Gamma_{\mu}, \Gamma_{\nu}\right]$, et cetera. Since the volume element acts trivially and Hodge duality is implemented by multiplication by the volume element, a basis for $\operatorname{End}(\Sigma)$ is given by $\left(\mathbb{1}, \Gamma_{\mu}, \Gamma_{\mu \nu}\right)$. Indeed, we have the following useful identities in $\operatorname{End}(\Sigma)$ for the other skewsymmetric products of the $\Gamma_{\mu}$ :

$$
\Gamma_{\mu \nu \rho}=-\frac{1}{2} \epsilon_{\mu \nu \rho \sigma \tau} \Gamma^{\sigma \tau}, \quad \Gamma_{\mu \nu \rho \tau}=\epsilon_{\mu \nu \rho \sigma \tau} \Gamma^{\tau} \quad \text { and } \quad \Gamma_{\mu \nu \rho \sigma \tau}=\epsilon_{\mu \nu \rho \sigma \tau} \mathbb{1} .
$$


Lemma 1. The following identities between gamma matrices hold:

$$
\begin{aligned}
\Gamma_{\mu} \Gamma^{\mu} & =5 \mathbb{1} \\
\Gamma_{\mu} \Gamma_{\nu} \Gamma^{\mu} & =-3 \Gamma_{\nu} \\
\Gamma_{\mu} \Gamma_{\nu \rho} \Gamma^{\mu} & =\Gamma_{\nu \rho}
\end{aligned}
$$

$$
\begin{aligned}
\frac{1}{2} \Gamma_{\mu \nu} \Gamma^{\mu \nu} & =-10 \mathbb{1} \\
\frac{1}{2} \Gamma_{\mu \nu} \Gamma_{\rho} \Gamma^{\mu \nu} & =-2 \Gamma_{\rho} \\
\frac{1}{2} \Gamma_{\mu \nu} \Gamma_{\rho \sigma} \Gamma^{\mu \nu} & =2 \Gamma_{\rho \sigma} .
\end{aligned}
$$

If $A \in \mathfrak{s o}(V)$, its action on $\Sigma$ is given by Clifford product with $\frac{1}{2} \omega_{A}$ :

$$
A s=\frac{1}{2} \omega_{A} \cdot s=\frac{1}{4} A^{\mu \nu} \Gamma_{\mu \nu} s .
$$

Let $\Delta$ denote a one-dimensional quaternionic (right) vector space, which we think as a two-dimensional complex vector space. We can similarly think of $\Sigma$ as a four-dimensional complex vector space with a quaternionic structure, so that the (complex) tensor product $\Sigma \otimes \mathbb{C} \Delta$ has a real structure and hence it is the complexification of a real eight-dimensional representation we denote by $S$. As a real vector space, $S$ is just $\Sigma$ when we restrict scalars

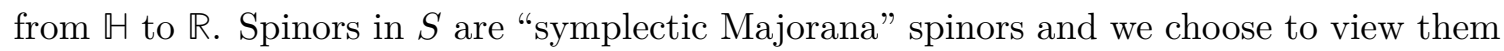
as pairs $s^{A} \in \Sigma$, where $A=1,2$, subject to a symplectic Majorana condition which uses the symplectic structures on $\Sigma$ and $\Delta$, respectively, and which we will write presently. Let us normalise $\epsilon_{A B}$ so that $\epsilon_{12}=+1$ and $\epsilon^{12}=+1$. We raise and lower indices with $\epsilon$ according to the conventions: $\epsilon_{A B} X^{B}{ }_{C}=X_{A C}$ and $\epsilon^{A B} X_{A C}=X^{B}{ }_{C}$, et cetera. The symplectic Majorana condition on the pair $s^{A}$ is such that

$$
\left(s^{A}\right)^{*}=\epsilon_{A B} \mathrm{~B} s^{B},
$$

where B obeys

$$
\mathrm{B} \Gamma_{\mu}=\Gamma_{\mu}^{*} \mathrm{~B}
$$

and normalised to $\mathrm{B}^{\dagger} \mathrm{B}=\mathbb{1}$.

It follows that the gamma matrices preserve $S$, hence so does the 16-dimensional real associative subalgebra $\mathbb{R} \Gamma \subset \operatorname{End}_{\mathbb{C}}(\Sigma)$ they generate. Note that this algebra is simply the image of $C \ell(V)$ under the representation on $\Sigma$. We denote by $\operatorname{End}_{\mathbb{H}} \Delta$ the real associative subalgebra of End $\mathbb{C} \Delta$ which preserves the quaternionic structure on $\Delta$. As a real vector space, $\operatorname{End}_{\mathfrak{H}} \Delta=\mathbb{R} \mathbb{1} \oplus \mathfrak{s p}(1)$. If we use the symplectic form to lower indices and identify $\Delta^{*} \cong \Delta$, we also identify $\operatorname{End}_{\mathbb{C}} \Delta \cong \otimes^{2} \Delta=\odot^{2} \Delta \oplus \wedge^{2} \Delta$. Under this identification, $\mathfrak{s p}(1)$ is the quaternionic structure-preserving part of $\odot^{2} \Delta$ and $\mathbb{R} \mathbb{1} \cong \mathbb{R} \epsilon$ is that of $\wedge^{2} \Delta$. We can identify $\operatorname{End}_{\mathbb{R}}(S)$ as the real subalgebra of $\operatorname{End}\left(\Sigma \otimes_{\mathbb{C}} \Delta\right)$ which preserves $S$, and it follows from the discussion above that $\operatorname{End}_{\mathbb{R}}(S)=\mathbb{R} \Gamma \otimes(\mathbb{R} \oplus \mathfrak{\mathbb { s }}(1))$. The inclusion in one direction is clear; the other then follows by a dimension count.

The symplectic structure $C$ on $\Sigma$ is real and satisfies

$$
\mathrm{C} \Gamma_{\mu}=\Gamma_{\mu}^{T} \mathrm{C} \Longrightarrow \mathrm{C} \Gamma_{\mu \nu}=-\Gamma_{\mu \nu}^{T} \mathrm{C} .
$$

We define for $s_{1}^{A}, s_{2}^{A} \in \Sigma$,

$$
\bar{s}_{1}^{A} s_{2}^{B}:=\left(s_{1}^{A}\right)^{T} \mathrm{C} s_{2}^{B} .
$$


Lemma 2. The following identities hold for all $s_{1}^{A}, s_{2}^{A} \in \Sigma$ :

1. $\bar{s}_{1}^{A} s_{2}^{B}=-\bar{s}_{2}^{B} s_{1}^{A}$,

2. $\bar{s}_{1}^{A} \Gamma_{\mu} s_{2}^{B}=-\bar{s}_{2}^{B} \Gamma_{\mu} s_{1}^{A}$,

3. $\bar{s}_{1}^{A} \Gamma_{\mu \nu} s_{2}^{B}=\bar{s}_{2}^{B} \Gamma_{\mu \nu} s_{1}^{A}$.

This allows us to define a symmetric inner product on $S$ :

$$
\left\langle s_{1}, s_{2}\right\rangle:=\epsilon_{A B} \bar{s}_{1}^{A} s_{2}^{B} .
$$

It follows from Lemma 2 that for all $v \in V$,

$$
\left\langle s_{1}, v \cdot s_{2}\right\rangle=\left\langle v \cdot s_{1}, s_{2}\right\rangle,
$$

and from this it follows that it is $\mathfrak{s o}(V)$-invariant:

$$
\left\langle A s_{1}, s_{2}\right\rangle=-\left\langle s_{1}, A s_{2}\right\rangle,
$$

for all $A \in \mathfrak{s o}(V)$.

There are a number of bilinears we can make from spinors. If $s \in S$, then we define

$$
\mu_{s}:=\epsilon_{A B} \bar{s}^{A} s^{B}, \quad \kappa_{s}^{\mu}:=\epsilon_{A B} \bar{s}^{A} \Gamma^{\mu} s^{B} \quad \text { and } \quad \omega_{s, \mu \nu}^{A B}=\bar{s}^{A} \Gamma_{\mu \nu} s^{B} .
$$

The map $\kappa: S \rightarrow V$ sending $s \mapsto \kappa_{s}$ defines the Dirac current of $s$ to be the unique vector $\kappa_{s} \in V$ such that for all $v \in V$,

$$
\eta\left(\kappa_{s}, v\right)=\langle s, v \cdot s\rangle .
$$

Similarly, $\mu_{s}=\langle s, s\rangle$ more invariantly and also for all $v, w \in V$,

$$
\omega_{s}^{A B}(v, w)=\frac{1}{2} \bar{s}^{A}[v, w] \cdot s^{B},
$$

where $[v, w]$ is the Clifford commutator. The following is the result of a calculation in an explicit realisation.

Lemma 3 (Reality conditions). For s symplectic Majorana, it follows that $\mu_{s}$ and $\kappa_{s}$ are real, whereas $\left(\omega_{s}^{11}\right)^{*}=\omega_{s}^{22}$ and $\left(\omega_{s}^{12}\right)^{*}=-\omega_{s}^{12}$.

The proof of the following Fierz identities is routine. We simply remark that $\operatorname{tr} \mathbb{1}=4$ since we are working in a four-dimensional complex vector space (with a quaternionic structure).

Lemma 4 (Fierz identities). For all $s_{1}^{A}, s_{2}^{B} \in \Sigma$,

$$
s_{1}^{A} \bar{s}_{2}^{B}=-\frac{1}{4} \bar{s}_{1}^{A} s_{2}^{B} \mathbb{1}-\frac{1}{4} \bar{s}_{1}^{A} \Gamma^{\mu} s_{2}^{B} \Gamma_{\mu}-\frac{1}{8} \bar{s}_{1}^{A} \Gamma^{\mu \nu} s_{2}^{B} \Gamma_{\mu \nu} .
$$

In particular, taking $s_{1}=s_{2}=s$ we arrive at

$$
s^{A-\bar{s}^{B}}=-\frac{1}{8} \epsilon^{A B} \mu_{s} \mathbb{1}-\frac{1}{8} \epsilon^{A B} \kappa_{s}-\frac{1}{4} \omega_{s}^{A B},
$$

where we define $\omega_{s}^{A B}=\frac{1}{2} \omega_{s, \mu \nu}^{A B} \Gamma^{\mu \nu}$. 
From this latter Fierz identity there follow some useful relations between the bilinears $\mu_{s}, \kappa_{s}$ and $\omega_{s}^{A B}$ defined above.

Lemma 5. Let $s \in S$ and let $\mu, \kappa$ and $\omega^{A B}$ be the corresponding bilinears. Then they satisfy the following relations:

(i) $\kappa \cdot s^{A}=\mu s^{A}$

(ii) $3 \mu s^{C}+\epsilon_{A B} \omega^{C A} \cdot s^{B}=0$

(iii) $\omega^{(A B} \cdot s^{C)}=0$

(iv) $\omega^{A B}(\kappa,-)=0$

Proof. As a result of the Fierz identity (2.23) we find that

$$
\begin{aligned}
\mu s^{C} & =\epsilon_{A B} \bar{s}^{A} s^{B} s^{C} \\
& =\epsilon_{A B} s^{C} \bar{s}^{A} s^{B} \\
& =\epsilon_{A B}\left(-\frac{1}{8} \epsilon^{C A} \mu \mathbb{1}-\frac{1}{8} \epsilon^{C A} \kappa-\frac{1}{4} \omega^{C A}\right) \cdot s^{B} \\
& =\frac{1}{8} \mu s^{C}+\frac{1}{8} \kappa \cdot s^{C}-\frac{1}{4} \epsilon_{A B} \omega^{C A} \cdot s^{B}
\end{aligned}
$$

or, equivalently,

$$
\frac{7}{8} \mu s^{C}-\frac{1}{8} \kappa \cdot s^{C}+\frac{1}{4} \epsilon_{A B} \omega^{C A} \cdot s^{B}=0 .
$$

Similarly, by calculating $\kappa \cdot s^{C}$ and using the Fierz identity (2.23), we arrive at

$$
\frac{5}{8} \mu s^{C}-\frac{11}{8} \kappa \cdot s^{C}-\frac{1}{4} \epsilon_{A B} \omega^{C A} \cdot s^{B}=0 .
$$

Adding equations (2.25) and (2.26), we obtain (i) and, plugging (i) back into either of those two equations, we obtain (ii). To obtain (iii), we calculate $\omega^{A B} \cdot s^{C}$ again using the Fierz identity (2.23), obtaining

$$
\omega^{A B} \cdot s^{C}+\frac{1}{2} \omega^{C A} \cdot s^{B}+\frac{5}{4} \epsilon^{A C} \mu s^{B}+\frac{1}{4} \epsilon^{A C} \kappa \cdot s^{B}=0 .
$$

Using (i), we rewrite this as

$$
\omega^{A B} \cdot s^{C}+\frac{1}{2} \omega^{C A} \cdot s^{B}+\frac{3}{2} \epsilon^{A C} \mu s^{B}=0 .
$$

This equation decomposes into two equations by (skew-)symmetrising in $A C$. The skewsymmetric component

$$
\omega^{B[A} \cdot s^{C]}+\frac{3}{2} \epsilon^{A C} \mu s^{B}=0
$$

vanishes identically by (ii), whereas the symmetric component

$$
\frac{1}{2} \omega^{B A} \cdot s^{C}+\frac{1}{2} \omega^{B C} \cdot s^{A}+\frac{1}{2} \omega^{C A} \cdot s^{B}=0
$$


is precisely (iii). Finally, to obtain (iv), we notice that for any $w \in V$,

$$
\begin{aligned}
\omega^{A B}(\kappa, w) & =\frac{1}{2} \bar{s}^{A}[\kappa, w] \cdot s^{B} \\
& =\frac{1}{2} \bar{s}^{A} \kappa \cdot w \cdot s^{B}-\frac{1}{2} \bar{s}^{A} w \cdot \kappa \cdot s^{B} \\
& =\frac{1}{2} \overline{\kappa \cdot s}{ }^{A} w \cdot s^{B}-\frac{1}{2} \bar{s}^{A} w \cdot \kappa \cdot s^{B},
\end{aligned}
$$

which vanishes by (i) and where we have used that:

$$
\bar{s}^{A} \kappa=\left(s^{A}\right)^{T} C \kappa=\left(s^{A}\right)^{T} \kappa^{T} C=\left(\kappa \cdot s^{A}\right)^{T} C=\overline{\kappa \cdot s}{ }^{A} .
$$

It follows from (ii) and (iii) in Lemma 5 that $\omega^{A B} \cdot s^{C}=-\mu\left(s^{A} \epsilon^{B C}+s^{B} \epsilon^{A C}\right)=$ $-2 \mu s^{(A} \epsilon^{B) C}$. In addition, it follows from (i) that $\eta(\kappa, \kappa)=\mu^{2} \geq 0$, so that $\kappa$ is causal: timelike if $\langle s, s\rangle \neq 0$ and null if $\langle s, s\rangle=0$, and it follows from (iv) that the elements of $\mathfrak{s o}(V)$ corresponding to $\omega^{A B}$ leave $\kappa$ invariant. We also have $\omega_{\mu \nu}^{A B} \omega_{A B}^{\mu \nu}=6 \mu^{2} \geq 0$ by (i), (ii), and a further application of the Fierz identity. There are other identities which can be derived, such as

$$
\omega_{[\mu \nu}^{A B} \kappa_{\rho]}=-\frac{1}{6} \mu \epsilon_{\mu \nu \rho \sigma \tau} \omega^{A B \sigma \tau} .
$$

The 2-forms $\omega^{A B}$ associated with $s$ define spinor endomorphisms

$$
\hat{\omega}^{A B}:=\frac{1}{4} \omega_{\mu \nu}^{A B} \Gamma^{\mu \nu},
$$

whose commutators satisfy:

$$
\left[\hat{\omega}^{12}, \hat{\omega}^{11}\right]=\mu \hat{\omega}^{11}, \quad\left[\hat{\omega}^{12}, \hat{\omega}^{22}\right]=-\mu \hat{\omega}^{22} \quad \text { and } \quad\left[\hat{\omega}^{11}, \hat{\omega}^{22}\right]=-2 \mu \hat{\omega}^{12},
$$

defining, for $\mu \neq 0$, the complex Lie algebra $\mathfrak{s l}(2, \mathbb{C})$. However, the symplectic Majorana condition on $s$ picks out a real form corresponding to the real 2 -forms; using Lemma 3 , this is the real span of the following spinor endomorphisms:

$$
L_{1}:=\frac{1}{2}\left(\hat{\omega}^{11}+\hat{\omega}^{22}\right), \quad L_{2}:=\frac{i}{2}\left(\hat{\omega}^{11}-\hat{\omega}^{22}\right) \quad \text { and } \quad L_{3}:=i \hat{\omega}^{12} .
$$

These satisfy

$$
\left[L_{i}, L_{j}\right]=\mu \epsilon_{i j k} L_{k},
$$

for $i, j, k=1,2,3$. In summary we have proved the following.

Proposition 6. Let $s \in S$ and $\mu, \kappa$ and $\omega^{A B}$ be the corresponding bilinears. Then the 2-forms $\omega^{A B}$ define a Lie subalgebra of the stabiliser of $\kappa$ in $\mathfrak{s o}(V)$ under the isomorphism $\mathfrak{s o}(V) \cong \wedge^{2} V$. If $\kappa$ is null (equivalently, $\mu=0$ ), this subalgebra is abelian and if $\kappa$ is timelike, the subalgebra is isomorphic to $\mathfrak{s p}(1)$. 


\subsection{The Poincaré superalgebra}

The Poincaré algebra $\mathfrak{p}(V)$ is the Lie algebra of isometries of $(V, \eta)$. It is a 15-dimensional $\mathbb{Z}$-graded Lie algebra with underlying vector space

$$
\mathfrak{p}(V)=\mathfrak{p}_{-2} \oplus \mathfrak{p}_{0}=V \oplus \mathfrak{s o}(V)
$$

and Lie brackets

$$
[A, B]=A B-B A \quad[A, v]=A v \quad \text { and } \quad[v, w]=0
$$

for all $v, w \in V$, and $A, B \in \mathfrak{s o}(V)$.

The $d=5$ Poincaré superalgebra $\mathfrak{s}(V)$ is the minimal superalgebra extending the Poincaré algebra of $V$. In fact, the even Lie algebra $\mathfrak{s}_{\overline{0}}=\mathfrak{p}(V)$ is the Poincaré algebra. The odd subspace $\mathfrak{s}_{\overline{1}}=S$, the real eight-dimensional Clifford module $S$. The underlying vector space of the $\mathbb{Z}$-graded Lie superalgebra $\mathfrak{s}(V)$ is

$$
\mathfrak{s}(V)=\mathfrak{s}_{-2} \oplus \mathfrak{s}_{-1} \oplus \mathfrak{s}_{0}=V \oplus S \oplus \mathfrak{s o}(V)
$$

and Lie brackets given by those in (2.39) and

$$
[A, s]=\frac{1}{2} \omega_{A} \cdot s \quad[v, s]=0 \quad \text { and } \quad[s, s]=\kappa_{s}
$$

for all $s \in S, v \in V$ and $A \in \mathfrak{s o}(V)$. In the remainder of the paper, we will denote the Poincaré superalgebra simply by $\mathfrak{s}$, leaving $V$ implicit in the notation.

\section{Spencer cohomology}

In this section we describe our calculation of the Spencer cohomology of the $d=5$ Poincare superalgebra.

\subsection{Lie algebra cohomology}

We start by briefly recalling the Chevalley-Eilenberg complex computing the cohomology of a (finite-dimensional) Lie algebra $\mathfrak{g}$ relative to a module $\mathfrak{M}$. Let $G$ be the simply-connected Lie group with Lie algebra $\mathfrak{g}$. The exterior derivative of a left-invariant differential form on $G$ is itself left-invariant and hence the left-invariant forms on $G$ define a sub-complex of the de Rham complex of $G$. Furthermore, since a left-invariant form is uniquely determined by its value at the identity, we may localise the sub-complex of left-invariant forms at the identity, resulting in a differential complex $\left(C^{\bullet}(\mathfrak{g})=\wedge^{\bullet} \mathfrak{g}^{*}, \partial\right)$ known as the ChevalleyEilenberg complex. Its cohomology $H^{\bullet}(\mathfrak{g})$ is the Lie algebra cohomology of $\mathfrak{g}$ or, more precisely, the Lie algebra cohomology of $\mathfrak{g}$ with values in the trivial module $\mathbb{R}$.

Let $\rho: \mathfrak{g} \rightarrow \mathfrak{g l}(\mathfrak{M})$ be a representation of $\mathfrak{g}$ and let us also denote by $\rho$ the corresponding representation of the simply-connected $G$. Letting $L_{g}: G \rightarrow G$ denote the left multiplication by $g \in G$, we may consider the $G$-equivariant $\mathfrak{M}$-valued differential forms on $G$ : $\mathfrak{M}$ valued differential forms $\omega$ which obey $L_{g}^{*} \omega=\rho(g) \circ \omega$ for all $g \in G$. Every such $\omega$ localises at the identity to a linear map $\wedge^{\bullet} \mathfrak{g} \rightarrow \mathfrak{M}$. The space $C^{\bullet}(\mathfrak{g}, \mathfrak{M}):=\mathfrak{M} \otimes \wedge^{\bullet} \mathfrak{g}^{*}$ of such maps 
becomes a complex under the Chevalley-Eilenberg differential $\partial: C^{k}(\mathfrak{g}, \mathfrak{M}) \rightarrow C^{k+1}(\mathfrak{g}, \mathfrak{M})$ defined by extending linearly the linear map

$$
\partial(m \otimes \omega)=\partial m \wedge \omega+m \otimes \partial \omega
$$

for $m \in \mathfrak{M}$ and $\omega \in \wedge^{k} \mathfrak{g}^{*}$, where $\partial \omega$ is induced, as before, by the de Rham differential and $\partial m: \mathfrak{g} \rightarrow \mathfrak{M}$ is defined by $\partial m(X)=\rho(X) m$ for all $X \in \mathfrak{g}$. The cohomology of this complex is denoted $H^{\bullet}(\mathfrak{g}, \mathfrak{M})$ and is called the Lie algebra cohomology of $\mathfrak{g}$ with values in the module $\mathfrak{M}$.

The preceding discussion extends to the case of $\mathfrak{g}$ a Lie superalgebra and $\mathfrak{M}$ a module, except that now $\wedge$ is taken in the super-sense (i.e., symmetric on odd elements) and we must insert the relevant Koszul signs. Explicitly, the Chevalley-Eilenberg differential $\partial$ : $C^{k}(\mathfrak{g}, \mathfrak{M}) \rightarrow C^{k+1}(\mathfrak{g}, \mathfrak{M})$ in low degree is given by

$$
\begin{aligned}
& \partial: C^{0}(\mathfrak{g}, \mathfrak{M}) \rightarrow C^{1}(\mathfrak{g}, \mathfrak{M}) \\
& \partial m(X)=\rho(X) m, \\
& \partial: C^{1}(\mathfrak{g}, \mathfrak{M}) \rightarrow C^{2}(\mathfrak{g}, \mathfrak{M}) \\
& \partial \varphi(X, Y)=\rho(X) \varphi(Y)-(-1)^{x y} \rho(Y) \varphi(X)-\varphi([X, Y]), \\
& \partial: C^{2}(\mathfrak{g}, \mathfrak{M}) \rightarrow C^{3}(\mathfrak{g}, \mathfrak{M}) \\
& \partial \varphi(X, Y, Z)=\rho(X) \varphi(Y, Z)+(-1)^{x(y+z)} \rho(Y) \varphi(Z, X)+(-1)^{z(x+y)} \rho(Z) \varphi(X, Y) \\
& -\varphi([X, Y], Z)-(-1)^{x(y+z)} \varphi([Y, Z], X)-(-1)^{z(x+y)} \varphi([Z, X], Y),
\end{aligned}
$$

where $x, y, z$ denote the parity of elements $X, Y, Z$ of $\mathfrak{g}$.

If we take $\mathfrak{M}=\mathfrak{g}$ to be the adjoint representation, with $\rho(X) Y=[X, Y]$, then $H^{\bullet}(\mathfrak{g}, \mathfrak{g})$ has the following interpretation in low degree:

- $H^{0}(\mathfrak{g}, \mathfrak{g})$ is isomorphic to the centre of $\mathfrak{g}$;

- $H^{1}(\mathfrak{g}, \mathfrak{g})$ is the quotient of the derivations of $\mathfrak{g}$ by the inner derivations; and

- $H^{2}(\mathfrak{g}, \mathfrak{g})$ are the infinitesimal deformations of $\mathfrak{g}$.

In this paper we concentrate on a refinement of this latter cohomology group for the case of graded Lie superalgebras. Rather than discuss this in full generality, let us already specialise to the Poincaré superalgebra defined in section 2.2.

\subsection{The Spencer complex}

Let $\mathfrak{s}_{-}:=\mathfrak{s}_{-1} \oplus \mathfrak{s}_{-2}$ denote the negative-degree subalgebra of the Poincaré superalgebra. It is a Lie superalgebra in its own right and the restriction of the adjoint action of $\mathfrak{s}$ to $\mathfrak{s}_{-}$ makes $\mathfrak{s}$ into a module of $\mathfrak{s}_{-}$. We define $C^{p}\left(\mathfrak{s}_{-} ; \mathfrak{s}\right)=\operatorname{Hom}\left(\wedge^{p} \mathfrak{s}_{-}, \mathfrak{s}\right)$, where $\wedge^{p}$ is taken in the super-sense. The direct sum $C^{\bullet}:=\bigoplus_{p} C^{p}\left(\mathfrak{s}_{-} ; \mathfrak{s}\right)$ becomes a differential complex relative to the Chevalley-Eilenberg differential of $\mathfrak{s}_{-}$relative to its module $\mathfrak{s}$. We extend the $\mathbb{Z}$-grading of $\mathfrak{s}$ to $C^{\bullet}$ in the natural way and, since $\mathfrak{s}$ is $\mathbb{Z}$-graded, the differential has degree 0 . Therefore the Chevalley-Eilenberg complex decomposes into subcomplexes of a fixed degree: we let 
$C^{d, p}\left(\mathfrak{s}_{-} ; \mathfrak{s}\right)$ denote the $p$-cochains of degree $d$ and $\partial: C^{d, p}\left(\mathfrak{s}_{-} ; \mathfrak{s}\right) \rightarrow C^{d, p+1}\left(\mathfrak{s}_{-} ; \mathfrak{s}\right)$ denote the restriction of the Chevalley-Eilenberg differential. We are interested in calculating $H^{2,2}\left(\mathfrak{s}_{-} ; \mathfrak{s}\right)$. The relevant spaces of cochains are:

$$
\begin{aligned}
& C^{2,1}\left(\mathfrak{s}_{-} ; \mathfrak{s}\right)=\operatorname{Hom}(V, \mathfrak{s o}(V)) \\
& C^{2,2}\left(\mathfrak{s}_{-} ; \mathfrak{s}\right)=\operatorname{Hom}\left(\wedge^{2} V, V\right) \oplus \operatorname{Hom}(V \otimes S, S) \oplus \operatorname{Hom}\left(\odot^{2} S, \mathfrak{s o}(V)\right) \\
& C^{2,3}\left(\mathfrak{s}_{-} ; \mathfrak{s}\right)=\operatorname{Hom}\left(\odot^{2} S \otimes V, V\right) \oplus \operatorname{Hom}\left(\odot^{3} S, S\right),
\end{aligned}
$$

and the differential can be read off from equations (3.3) and (3.4) above, with $\rho(X) Y=$ $[X, Y]$ for $X \in \mathfrak{s}_{-}$and $Y \in \mathfrak{s}$.

As usual, the Spencer differential $\partial: C^{2,1}\left(\mathfrak{s}_{-} ; \mathfrak{s}\right) \rightarrow C^{2,2}\left(\mathfrak{s}_{-} ; \mathfrak{s}\right)$ is injective and hence $H^{2,1}\left(\mathfrak{s}_{-} ; \mathfrak{s}\right)=0$. Moreover, $H^{2,2}\left(\mathfrak{s}_{-} ; \mathfrak{s}\right) \cong \mathscr{H}^{2,2}$, where

$$
\mathscr{H}^{2,2}=\operatorname{ker} \pi_{1} \cap Z^{2,2},
$$

where $Z^{2,2}$ is the space of Spencer cocycles in $C^{2,2}\left(\mathfrak{s}_{-} ; \mathfrak{s}\right)$ and $\pi_{1}: C^{2,2}\left(\mathfrak{s}_{-} ; \mathfrak{s}\right) \rightarrow$ $\operatorname{Hom}\left(\wedge^{2} V, V\right)$ is the natural projection onto the first summand. In other words, cohomology classes in $H^{2,2}\left(\mathfrak{s}_{-} ; \mathfrak{s}\right)$ are in bijective correspondence with normalised cocycles $\beta+\gamma \in Z^{2,2}$, where $\beta: V \otimes S \rightarrow S$ and $\gamma: \odot^{2} S \rightarrow \mathfrak{s o}(V)$.

There are two components to the cocycle condition: one in $\operatorname{Hom}\left(\odot^{2} S \otimes V, V\right)$ :

$$
\gamma(s, s) v+2[s, \beta(v, s)]=0,
$$

and one in $\operatorname{Hom}\left(\odot^{3} S, S\right)$

$$
\gamma(s, s) s+\beta([s, s], s)=0,
$$

for all $v \in V$ and $s \in S$.

\subsection{Solution of the first cocycle condition}

We define $\beta_{v} \in \operatorname{End}(S)$ by $\beta_{v}(s)=\beta(v, s)$ for $v \in V$ and $s \in S$. We let $\beta_{\mu}:=\beta_{\boldsymbol{e}_{\mu}}$ and parametrise $\beta_{\mu}$ as follows:

$$
\left(\beta_{\mu} s\right)^{B}=A_{\mu} s^{B}+B_{\mu}{ }^{B}{ }_{C} s^{C}+C_{\mu \nu} \Gamma^{\nu} s^{B}+D_{\mu \nu}{ }^{B}{ }_{C} \Gamma^{\nu} s^{C}+\frac{1}{2} E_{\mu \nu \rho} \Gamma^{\nu \rho} s^{B}+\frac{1}{2} F_{\mu \nu \rho}{ }_{C} \Gamma^{\nu \rho} s^{C},
$$

where $B_{\mu}, D_{\mu \nu}, F_{\mu \nu \rho} \in \mathfrak{s p}(1)$. In particular, with all lowered $\Delta$-indices, these components are symmetric in those indices. The first cocycle condition (3.7) becomes

$$
\gamma(s, s)_{\mu \nu}+2 \epsilon_{A B} \bar{s}^{A} \Gamma_{\mu}\left(\beta_{\nu} s\right)^{B}=0 .
$$

The skew-symmetric part in $\mu \nu$ expresses $\gamma(s, s)$ in terms of $\beta$, whereas the symmetric part constrains $\beta$.

Lemma 7 (First cocycle condition). The solution of the first cocycle condition (3.7) is given by

$$
\begin{aligned}
\left(\beta_{\mu} s\right)^{B} & =B_{\mu}{ }^{B}{ }_{C} s^{C}+C_{\mu \nu} \Gamma^{\nu} s^{B}+\frac{1}{5} D^{B}{ }_{C} \Gamma_{\mu} s^{C}+\frac{1}{2} E_{\mu \nu \rho} \Gamma^{\nu \rho} s^{B}+\frac{1}{4} F^{\rho B}{ }_{C} \Gamma_{\mu \rho} s^{C} \\
\gamma(s, s)_{\mu \nu} & =2 \mu C_{\mu \nu}-\frac{2}{5} D_{A B} \omega_{\mu \nu}^{A B}+2 E_{\mu \nu \rho} \kappa^{\rho}+\frac{1}{4} \epsilon_{\mu \nu \rho}{ }^{\sigma \tau} F_{A B}^{\rho} \omega_{\sigma \tau}^{A B},
\end{aligned}
$$

for some $C \in \wedge^{2} V$ and $E \in \wedge^{3} V$. 
Proof. Symmetrising the first cocycle condition in $\mu \nu$ results in

$$
0=\epsilon_{A B} \bar{s}^{A}\left(\Gamma_{\mu}\left(\beta_{\nu} s\right)^{B}+\Gamma_{\nu}\left(\beta_{\mu} s\right)^{B}\right),
$$

which expands to

$$
\begin{aligned}
0= & 2 \kappa_{(\mu} A_{\nu)}+2 \mu C_{(\mu \nu)}+D_{\mu}{ }^{\rho} A B \omega_{\nu \rho}^{A B}+D_{\nu}{ }^{\rho} A B \omega_{\mu \rho}^{A B}+2 E_{(\mu \nu) \rho} \kappa^{\rho} \\
& -\frac{1}{4} \epsilon_{\mu \rho \sigma}{ }^{\tau \xi} F_{\nu}{ }^{\rho \sigma}{ }_{A B} \omega_{\tau \xi}^{A B}-\frac{1}{4} \epsilon_{\nu \rho \sigma}{ }^{\tau \xi} F_{\mu}{ }^{\rho \sigma}{ }_{A B} \omega_{\tau \xi}^{A B} .
\end{aligned}
$$

Since this equation holds for all $s \in S$, the terms in $\mu, \kappa^{\mu}$ and $\omega_{\mu \nu}^{A B}$ must separately vanish. The $\mu$-term simply says that $C_{(\mu \nu)}=0$, so that $C \in \wedge^{2} V$. (We do not distinguish between $V$ and $V^{*}$.) The $\kappa$-terms result in the equation

$$
0=E_{\mu \nu \rho}+E_{\nu \mu \rho}+\eta_{\mu \rho} A_{\nu}+\eta_{\nu \rho} A_{\mu}
$$

Adding the cyclic permutations of this equation and using that $E_{\mu \nu \rho}=-E_{\mu \rho \nu}$, we find that

$$
\eta_{(\mu \nu} A_{\rho)}=0 \Longrightarrow A_{\mu}=0
$$

and hence that $E_{\mu \nu \rho}=-E_{\nu \mu \rho}$, which says that $E \in \wedge{ }^{3} V$. Finally, the $\omega$-terms result in

$$
0=D_{\mu \xi} \eta_{\tau \nu}+D_{\nu \xi} \eta_{\tau \mu}-D_{\mu \tau} \eta_{\xi \nu}-D_{\nu \tau} \eta_{\xi \mu}-\frac{1}{2} \epsilon_{\mu \rho \sigma \tau \xi} F_{\nu}{ }^{\rho \sigma}-\frac{1}{2} \epsilon_{\nu \rho \sigma \tau \xi} F_{\mu}{ }^{\rho \sigma}
$$

where we have omitted the $\mathfrak{s p}(1)$-indices. Tracing with $\eta^{\mu \nu}$ we find

$$
D_{[\tau \xi]}=\frac{1}{4} \epsilon_{\tau \xi \nu \rho \sigma} F^{\nu \rho \sigma}
$$

whereas tracing with $\eta^{\nu \tau}$ yields

$$
5 D_{\mu \xi}-D \eta_{\mu \xi}+\frac{1}{2} \epsilon_{\mu \xi \rho \sigma \tau} F^{\tau \rho \sigma}=0,
$$

where we have introduced $D:=\eta^{\mu \nu} D_{\mu \nu}$. Breaking up into symmetric and skew-symmetric parts, we find

$$
D_{(\mu \xi)}=\frac{1}{5} \eta_{\mu \xi} D \quad \text { and } \quad D_{[\mu \xi]}=-\frac{1}{10} \epsilon_{\mu \xi \rho \sigma \tau} F^{\rho \sigma \tau} .
$$

Taking these equations together with equation (3.17), we conclude that $D_{\mu \nu}=\frac{1}{5} \eta_{\mu \nu} D$ and $\epsilon_{\mu \nu \rho \sigma \tau} F^{\rho \sigma \tau}=0$, so that the $\wedge^{3} V$ component of $F$ vanishes. Back into the $\omega$-equation (3.16), we find

$$
\epsilon_{\mu \rho \sigma \tau \xi} F_{\nu}^{\rho \sigma}+\epsilon_{\nu \rho \sigma \tau \xi} F_{\mu}^{\rho \sigma}=0 .
$$

Contracting with $\epsilon^{\nu \tau \xi \alpha \beta}$, we arrive at

$$
16 F_{\mu}^{\alpha \beta}+4 \delta_{\mu}^{\beta} F_{\nu}^{\nu \alpha}-4 \delta_{\mu}^{\alpha} F_{\nu}{ }^{\nu \beta}=0 \Longrightarrow F_{\mu \nu \rho}=\frac{1}{4}\left(\eta_{\mu \nu} F_{\rho}-\eta_{\mu \rho} F_{\nu}\right),
$$

where we have defined $F_{\mu}:=F^{\nu}{ }_{\nu \mu}$. Collecting these results together, we arrive at the expressions for $\beta$ and $\gamma$ in the statement of the lemma. 


\subsection{Solution of the second cocycle condition}

The second cocycle condition (3.8) becomes

$$
\frac{1}{4} \gamma(s, s)_{\mu \nu} \Gamma^{\mu \nu} s^{B}+\kappa^{\mu}\left(\beta_{\mu} s\right)^{B}=0,
$$

where $\gamma(s, s)_{\mu \nu}$ and $\left(\beta_{\mu} s\right)^{B}$ are given in Lemma 7 .

Lemma 8 (Second cocycle condition). The second cocycle condition reduces to

$$
D^{B}{ }_{C}=0, \quad B_{\mu}{ }^{B}{ }_{C}=-\frac{1}{2} F_{\mu}{ }^{B}{ }_{C} \quad \text { and } \quad E_{\mu \nu \rho}=\frac{1}{4} \epsilon_{\mu \nu \rho \sigma \tau} C^{\sigma \tau} .
$$

Proof. From the expressions for $\beta$ and $\gamma$ in Lemma 7, the second cocycle condition (3.22) can be written as

$$
\left(\mu \Theta^{B}{ }_{C}+\kappa^{\mu} \Psi_{\mu}{ }^{B} C+\frac{1}{2} \omega_{\mu \nu}^{A D} \Phi_{A D}^{\mu \nu} \delta_{C}^{B}\right) s^{C}=0,
$$

where we have introduced the shorthands

$$
\begin{aligned}
\Theta^{B}{ }_{C} & :=\frac{1}{2} C_{\mu \nu} \Gamma^{\mu \nu} \delta_{C}^{B}+\frac{1}{2} D^{B}{ }_{C}-\frac{1}{4} F_{\mu}{ }_{C} \Gamma^{\mu} \\
\Psi_{\mu}{ }^{B}{ }_{C} & :=E_{\mu \nu \rho} \Gamma^{\nu \rho} \delta_{C}^{B}+B_{\mu}{ }^{B}{ }_{C}+\frac{1}{4} F_{\mu}{ }^{B}{ }_{C}+C_{\mu \nu} \Gamma^{\nu} \delta_{C}^{B} \\
\Phi_{A B}^{\mu \nu} & :=\frac{1}{8} \epsilon^{\mu \nu \rho \sigma \tau} F_{\rho A B} \Gamma_{\sigma \tau} .
\end{aligned}
$$

We expand $\mu, \kappa$ and $\omega$ in equation (3.24) to obtain

$$
\left(\epsilon_{A B} \bar{s}^{A} s^{B} \Theta^{C}{ }_{D}+\epsilon_{A B} \bar{s}^{A} \Gamma^{\mu} s^{B} \Psi_{\mu}{ }^{C}{ }_{D}+\frac{1}{2} \bar{s}^{A} \Gamma_{\mu \nu} s^{B} \Phi_{A B}^{\mu \nu} \delta_{D}^{C}\right) s^{D}=0
$$

and we polarise away from the diagonal:

$$
\begin{aligned}
& \left(\epsilon_{A B} \bar{s}_{1}^{A} s_{2}^{B} \Theta^{C}{ }_{D}+\epsilon_{A B} \bar{s}_{1}^{A} \Gamma^{\mu} s_{2}^{B} \Psi_{\mu}{ }^{C}{ }_{D}+\frac{1}{2} \bar{s}_{1}^{A} \Gamma_{\mu \nu} s_{2}^{B} \Phi_{A B}^{\mu \nu} \delta_{D}^{C}\right) s_{3}^{D} \\
+ & \left(\epsilon_{A B} \bar{s}_{2}^{A} s_{3}^{B} \Theta^{C}{ }_{D}+\epsilon_{A B} \bar{s}_{2}^{A} \Gamma^{\mu} s_{3}^{B} \Psi_{\mu}{ }^{C}{ }_{D}+\frac{1}{2} \bar{s}_{2}^{A} \Gamma_{\mu \nu} s_{3}^{B} \Phi_{A B}^{\mu \nu} \delta_{D}^{C}\right) s_{1}^{D} \\
+ & \left(\epsilon_{A B} \bar{s}_{1}^{A} s_{3}^{B} \Theta^{C}{ }_{D}+\epsilon_{A B} \bar{s}_{1}^{A} \Gamma^{\mu} s_{3}^{B} \Psi_{\mu}{ }^{C}{ }_{D}+\frac{1}{2} \bar{s}_{1}^{A} \Gamma_{\mu \nu} s_{3}^{B} \Phi_{A B}^{\mu \nu} \delta_{D}^{C}\right) s_{2}^{D}=0
\end{aligned}
$$

where in the last line we have used the symmetry properties of Lemma 2. We write this as an endomorphism acting on $s_{3}^{D}$ :

$$
\begin{aligned}
& \left(\epsilon_{A B} \bar{s}_{1}^{A} s_{2}^{B} \Theta^{C}{ }_{D}+\epsilon_{A B} \bar{s}_{1}^{A} \Gamma^{\mu} s_{2}^{B} \Psi_{\mu}{ }^{C}{ }_{D}+\frac{1}{2} \bar{s}_{1}^{A} \Gamma_{\mu \nu} s_{2}^{B} \Phi_{A B}^{\mu \nu} \delta_{D}^{C}\right. \\
& \left.\quad+2 \epsilon_{A D} \Theta^{C}{ }_{B} s_{1}^{B} \bar{s}_{2}^{A} s_{3}^{D}+2 \epsilon_{A D} \Psi_{\mu}{ }^{C}{ }_{B} s_{1}^{B} \bar{s}_{2}^{A} \Gamma^{\mu}+\Phi_{A D}^{\mu \nu} s_{1}^{C} \bar{s}_{2}^{A} \Gamma_{\mu \nu}\right) s_{3}^{D}=0 .
\end{aligned}
$$

This is true for all $s_{3}$, hence the endomorphism in parenthesis has to vanish for all $s_{1}$ and $s_{2}$. Being symmetric in $s_{1}$ and $s_{2}$, it is uniquely characterised by its values on the diagonal, so we can simply take $s_{1}=s_{2}=s$ and rewrite the second cocycle condition as

$$
\begin{aligned}
\epsilon_{A B} \bar{s}^{A} s^{B} \Theta^{C}{ }_{D}+ & \epsilon_{A B} \bar{s}^{A} \Gamma^{\mu} s^{B} \Psi_{\mu}{ }^{C}{ }_{D}+\frac{1}{2} \bar{s}^{A} \Gamma_{\mu \nu} s^{B} \Phi_{A B}^{\mu \nu} \delta_{D}^{C} \\
& +2 \epsilon_{A D} \Theta^{C}{ }_{B} s^{B} \bar{s}^{A} s_{3}^{D}+2 \epsilon_{A D} \Psi_{\mu}{ }^{C}{ }_{B} s^{B} \bar{s}^{A} \Gamma^{\mu}+\Phi_{A D}^{\mu \nu} s^{C} \bar{s}^{A} \Gamma_{\mu \nu}=0 .
\end{aligned}
$$


We use the Fierz identity (2.23) in the last three terms and we collect terms in $\mu, \kappa$ and $\omega$ together, each of which must vanish (as an endomorphism of $S$ ) separately, resulting in three equations:

$$
\begin{array}{r}
5 \Theta^{C}{ }_{D}+\Psi_{\mu}{ }^{C}{ }_{D} \Gamma^{\mu}+\frac{1}{2} \epsilon^{A C} \Phi_{A D}^{\mu \nu} \Gamma_{\mu \nu}=0 \\
5 \Psi_{\mu}{ }^{C}{ }_{D}+\Theta^{C}{ }_{D} \Gamma_{\mu}+\Psi^{\nu C}{ }_{D} \Gamma_{\mu \nu}+\frac{1}{2} \epsilon^{A C} \Phi_{A D}^{\nu \rho} \Gamma_{\mu} \Gamma_{\nu \rho}=0
\end{array}
$$

and

$$
\Phi_{A B}^{\mu \nu} \delta_{D}^{C}+\frac{1}{2} \epsilon_{D(A} \Theta^{C}{ }_{B)} \Gamma^{\mu \nu}-\frac{1}{2} \Psi_{\rho}^{C}{ }_{(A} \epsilon_{B) D} \Gamma^{\mu \nu} \Gamma^{\rho}-\frac{1}{4} \delta_{(A}^{C} \Phi_{B) D}^{\rho \sigma} \Gamma^{\mu \nu} \Gamma_{\rho \sigma}=0 .
$$

We notice that the $\mu$-equation (3.30) is the Clifford trace of the $\kappa$-equation (3.31): indeed, contracting equation (3.31) on the right with $\Gamma^{\mu}$ we obtain equation (3.30). Therefore the $\mu$-equation is redundant and we concentrate on the other two equations.

The $\kappa$-equation can be rewritten as

$$
5 \Psi_{\mu A B}+\Theta_{A B} \Gamma_{\mu}+\Psi_{A B}^{\nu} \Gamma_{\mu \nu}+\frac{1}{2} \Phi_{A B}^{\nu \rho} \Gamma_{\mu} \Gamma_{\nu \rho}=0 .
$$

Upon substituting the expressions for $\Theta, \Psi$ and $\Phi$ into this equation, we first symmetrise in $A B$ (dropping the indices) and using the Clifford relations we arrive at

$$
5\left(B_{\mu}+\frac{1}{2} F_{\mu}\right)+\left(B^{\nu}+\frac{1}{2} F^{\nu}\right) \Gamma_{\mu \nu}+\frac{1}{2} D \Gamma_{\mu}=0,
$$

which results in

$$
D_{A B}=0 \quad \text { and } \quad B_{A B}^{\mu}=-\frac{1}{2} F_{A B}^{\mu} .
$$

If instead we skew-symmetrise in $A B$ we arrive at

$$
5\left(E_{\mu \nu \rho} \Gamma^{\nu \rho}+C_{\mu \nu} \Gamma^{\nu}\right)+\frac{1}{2} C_{\nu \rho} \Gamma^{\nu \rho} \Gamma_{\mu}+\left(E^{\nu \sigma \tau} \Gamma_{\sigma \tau}+C_{\rho}^{\nu \rho}\right) \Gamma_{\mu \nu}=0 .
$$

Contracting with $\Gamma^{\mu}$ on the right and simplifying we find

$$
3 C_{\mu \nu}=\epsilon_{\mu \nu \rho \sigma \tau} E^{\rho \sigma \tau}
$$

which can be inverted to write

$$
E_{\mu \nu \rho}=\frac{1}{4} \epsilon_{\mu \nu \rho \sigma \tau} C^{\sigma \tau}
$$

Re-inserting this back into equation (3.36), we find that it is identically satisfied.

One can check that the remaining equation (3.32) is identically satisfied.

In summary, we have proved the following

Theorem 9. As a module of $\mathfrak{s o}(V) \oplus \mathfrak{s p}(1)$, we have the following isomorphism:

$$
H^{2,2}\left(\mathfrak{s}_{-} ; \mathfrak{s}\right) \cong\left(\wedge^{2} V \otimes \wedge^{2} \Delta\right) \oplus\left(V \otimes \odot^{2} \Delta\right)
$$


where to a class $\left(C_{\mu \nu} \epsilon_{A B}, F_{A B}^{\mu}\right) \in H^{2,2}$ there corresponds the cocycle $(\beta, \gamma) \in \operatorname{Hom}(V \otimes$ $S, S) \oplus \operatorname{Hom}\left(\odot^{2} S, \mathfrak{s o}(V)\right)$ given by

$$
\begin{aligned}
& \beta(v, s)^{B}=\frac{1}{4} v \cdot C \cdot s^{B}-\frac{3}{4} C \cdot v \cdot s^{B}-\frac{1}{8} v \cdot F^{B}{ }_{C} \cdot s^{C}-\frac{3}{8} F^{B}{ }_{C} \cdot v \cdot s^{C} \\
& \gamma(s, s)_{\mu \nu}=2 \mu C_{\mu \nu}+\frac{1}{2} \kappa^{\rho} \epsilon_{\mu \nu \rho \sigma \tau} C^{\sigma \tau}+\frac{1}{4} \epsilon_{\mu \nu \rho}^{\sigma \tau} F_{A B}^{\rho} \omega_{\sigma \tau}^{A B} .
\end{aligned}
$$

This is not far from the naive dimensional reduction of the result in [14]: the self-dual 3 -form reduces dimensionally to a 2 -form $\left(C_{\mu \nu}\right)$ and a 3 -form $\left(E_{\mu \nu \rho}\right)$ which are related by Hodge duality and the $\mathfrak{s p}(1)$-valued 1-form gives rise to an $\mathfrak{s p}(1)$-valued 1-form $\left(F_{\mu}{ }_{B}\right)$ and an $\mathfrak{s p}(1)$-valued scalar, which is missing from the five-dimensional calculation. The precise behaviour of the Spencer cohomology under dimensional reduction lies beyond the scope of this paper and will be addressed in a separate paper.

\section{Zero-curvature equations}

In this section we interpret the cohomological calculations of the previous section geometrically. The first step is to re-interpret the Spencer complex geometrically and we do this in section 4.1, arriving at a connection $\mathscr{D}$ on spinor fields, whose curvature we calculate in section 4.3. The final aim of this section is to derive the conditions for maximal supersymmetry, which at least locally is tantamount to the flatness of $\mathscr{D}$. We do this in two steps: in section 4.4 we impose the vanishing of the Clifford trace of the curvature, which in the supergravity context often coincides with the bosonic field equations, and finally in section 4.5 we derive the conditions for vanishing curvature. In section 5 we will show that the $\mathscr{D}$-parallel spinor fields generate a Lie superalgebra at least when the curvature of $\mathscr{D}$ is Clifford-traceless and in section 6 we will study the geometries on which $\mathscr{D}$ is flat.

\subsection{Setup}

We shall fix a five-dimensional lorentzian spin manifold $(M, g)$ and let $V$ be as in section 2; that is, $V$ is a five-dimensional lorentzian vector space we may identify with $\mathbb{R}^{1,4}$. The spin bundle $\operatorname{Spin}(M)$ is a principal $\operatorname{Spin}(V)$-bundle which comes with a bundle morphism $\operatorname{Spin}(M) \rightarrow \mathrm{SO}(M)$ to the oriented orthonormal frame bundle, covering the identity and agreeing fibrewise with the standard 2-to-1 covering homomorphism $\operatorname{Spin}(V) \rightarrow \mathrm{SO}(V)$. The principal bundle $\mathrm{SO}(M)$ is a $G$-structure with $G=\mathrm{SO}(V)$ and therefore comes with a soldering form which restricts pointwise to a vector space isomorphism $T_{p} M \rightarrow V$. These isomorphisms assemble to a bundle isomorphism between $T M$ and the "fake tangent bundle" $\mathrm{SO}(M) \times{ }_{\mathrm{SO}(V)} V$, which is the associated vector bundle of $\mathrm{SO}(M)$ corresponding to the vector representation of $\mathrm{SO}(V)$. Using this construction, we may locally write the components of tensor fields on $M$ as if they were tensors on $V$ using the orthonormal basis on $V$. This may be equivalently viewed as working in a local orthonormal frame on $M$.

Since we are interested in spin manifolds, the relevant principal bundle is $\operatorname{Spin}(M)$. Any associated vector bundle to $\mathrm{SO}(M)$ can be interpreted as an associated vector bundle 
to $\operatorname{Spin}(M)$ via the bundle morphism $\operatorname{Spin}(M) \rightarrow \mathrm{SO}(M)$, but there are of course also associated vector bundles to $\operatorname{Spin}(M)$ which do not arise in this way: namely, those involving spinorial representations.

As in section 2.1, let $\Sigma$ denote one of the two inequivalent Clifford modules of $C \ell(V)$. It becomes a $\operatorname{Spin}(V)$-module by restriction. Let $\$:=\operatorname{Spin}(M) \times{ }_{\operatorname{Spin}(V)} \Sigma$ denote the corresponding spinor bundle. It is a complex rank-4 vector bundle with a quaternionic structure $J$ and an invariant symplectic inner product $(\sigma, \tau) \mapsto \bar{\sigma} \tau$ inherited from $\Sigma$. We introduce an auxiliary trivial ${ }^{3}$ complex rank-2 vector bundle $\mathscr{H} \cong M \times \Delta$. It too has a quaternionic structure $j$ and a symplectic inner product $(\cdot, \cdot)$ inherited from $\Delta$. We will make a global choice of symplectic frame $e_{1}, e_{2}$ for $\mathscr{H}$ such that $\left(e_{A}, e_{B}\right)=\epsilon_{A B}$, where $\epsilon_{A B}$ is as in section 2.1. On the tensor product bundle $\mathbb{S}_{\mathbb{C}}:=\$ \otimes \mathscr{H}$ we have an invariant real structure $J \otimes j$. Its real sub-bundle $\mathbb{S}$ is the real rank-8 vector bundle associated to $\operatorname{Spin}(M)$ via the representation $S$, whose complexification $S \otimes_{\mathbb{R}} \mathbb{C}=\Sigma \otimes_{\mathbb{C}} \Delta$. Any spinor section $s$ of $\mathbb{S}_{\mathbb{C}}$ or $\mathbb{S}$ may be expanded relative to the global frame $e_{A}$ as $s=s^{A} \otimes e_{A}$, where in the case of $\mathbb{S}$ the $s^{A}$ are subject to the reality condition (2.12). The symplectic inner products on $\$$ and $\mathscr{H}$ combine to an inner product on $\mathbb{S}_{\mathbb{C}}$ given by $\left\langle s_{1}, s_{2}\right\rangle=\epsilon_{A B} \bar{s}_{1}^{A} s_{2}^{B}$, which is real when restricted to $\mathbb{S}$. We shall refer to sections of $\mathbb{S}_{\mathbb{C}}$ as (symplectic) Dirac spinor fields and to sections of $\mathbb{S}$ as (symplectic) Majorana spinor fields.

In summary, with any representation $W$ of $\operatorname{Spin}(V)$ made out of $V$ and $S$ (via tensor product and taking duals) we can associate a vector bundle $\operatorname{Spin}(M) \times_{\operatorname{Spin}(V)} W$ in such a way that to any $\operatorname{Spin}(V)$-equivariant linear map $\varphi: W_{1} \rightarrow W_{2}$ between two such representations, we associate a corresponding bundle morphism $\operatorname{Spin}(M) \times_{\operatorname{Spin}(V)} W_{1} \rightarrow$ $\operatorname{Spin}(M) \times_{\operatorname{Spin}(V)} W_{2}$. Since the cochains in the generalised Spencer complex are $\operatorname{Spin}(V)$ modules and the differential is $\operatorname{Spin}(V)$-equivariant, we may interpret the Spencer complex as a complex of the associated vector bundles and, in particular, as a complex on their spaces of smooth sections, and similarly for its cohomology.

In this way, for example, the component $\beta$ of the Spencer cocycle in equation (3.40) can be interpreted as a section of the vector bundle associated to the representation $\operatorname{Hom}(V, \operatorname{End}(S))$, which is the bundle of one-forms with values in $\operatorname{End}(\mathbb{S})$. This is the bundle on whose space of sections the affine space of connections on $\mathbb{S}$ is modelled on and therefore we may understand $\beta$ as the difference between two such connections. The natural connection on $\mathbb{S}$ is the spin connection $\nabla$ - that is, the one induced from the lift to $\operatorname{Spin}(M)$ of the Ehresmann connection on $\mathrm{SO}(M)$ which induces the Levi-Civita connection on $T M$ - and therefore we may interpret the cocycle component $\beta$ as $\nabla-\mathscr{D}$ for some connection $\mathscr{D}$ on $\mathbb{S}$. The cocycle component $\beta$ depends on the additional geometric data parametrising the relevant Spencer cohomology, namely a two-form $C \in \Omega^{2}(M)$ and an $\mathfrak{s p}(1)$-valued one form $F \in \Omega^{1}(M, \mathfrak{s p}(1))$.

Thus let us define a (bosonic) background $(M, g, C, F)$ to be a spin manifold $(M, g)$ with the structures described above along with the forms $C \in \Omega^{2}(M)$ and $F \in$ $\Omega^{1}(M ; \mathfrak{s p}(1))$. It will be useful in places to view the components $F_{B}^{A}$ of $F$ in a symplec-

\footnotetext{
${ }^{3}$ It could be interesting to relax this condition, gauge the R-symmetry and introduce a connection on a possibly non-trivial R-symmetry bundle, but that lies beyond the scope of the present paper.
} 
tic frame for $\mathscr{H}$ as 1 -forms on $M$. The End(\$)-valued one-form $\beta$ corresponding to the Spencer cocycle in equation (3.40) is given by

$$
\beta_{X} s=\frac{1}{4} X \cdot C \cdot s-\frac{3}{4} C \cdot X \cdot s-\frac{1}{8} X \cdot F \cdot s-\frac{3}{8} F \cdot X \cdot s,
$$

where $s$ is a Majorana spinor field, $X$ is a vector field, · denotes both the Clifford multiplication of forms and the Clifford action of forms on spinor fields, and $F$ also acts via the $\mathfrak{s p}(1)$ action on $\Gamma(\mathbb{S})$ to the right. ${ }^{4}$

With the conventions chosen above, in components we have

$$
\left(\beta_{\mu} s\right)^{A}=\frac{1}{8} C^{\alpha \beta}\left(\epsilon_{\mu \alpha \beta \sigma \tau} \Gamma^{\sigma \tau}+8 \eta_{\mu \alpha} \Gamma_{\beta}\right) s^{A}+\frac{1}{4} F_{B}^{\alpha A}\left(\Gamma_{\mu \alpha}-2 \eta_{\mu \alpha}\right) s^{B} .
$$

We define the superconnection $\mathscr{D}$ on $\mathbb{S}$ by

$$
\mathscr{D}_{X} s=\nabla_{X} s-\beta_{X} s
$$

where $\nabla$ is (the spin lift of) the Levi-Civita connection, $X \in \mathfrak{X}(M)$ and $s \in \Gamma(\mathbb{S})$. In components,

$$
\left(\mathscr{D}_{\mu} s\right)^{A}=\nabla_{\mu} s^{A}-\frac{1}{8} C^{\alpha \beta}\left(\epsilon_{\mu \alpha \beta \sigma \tau} \Gamma^{\sigma \tau}+8 \eta_{\mu \alpha} \Gamma_{\beta}\right) s^{A}-\frac{1}{4} F_{B}^{\alpha A}\left(\Gamma_{\mu \alpha}-2 \eta_{\mu \alpha}\right) s^{B} .
$$

Let us remark that, contrary to what one might have suspected, the $\mathfrak{s p}(1)$-valued oneform $F$ does not correspond to the difference between two connections on the auxiliary bundle $\mathscr{H}$. If that were the case, the term $\frac{1}{4} F^{\alpha A}{ }_{B} \Gamma_{\mu \alpha}$ in the expression for $\mathscr{D}$ would be absent, while its presence suggests a mixing of local Lorentz and R-symmetries.

Definition 10. A Killing spinor (field) on a background $(M, g, C, F)$ is a spinor field $s \in \Gamma(\mathbb{S})$ which is parallel with respect to the superconnection $\mathscr{D}$; that is, if it satisfies the Killing spinor equation

$$
\nabla s=\beta s .
$$

A background $(M, g, C, F)$ is supersymmetric if it admits a Killing spinor, and maximally supersymmetric if its space of Killing spinors has maximal dimension.

The notion of "maximal dimension" in the definition above arises because a set of linearly independent sections has linearly independent values at all points; hence such a set has size at most $\operatorname{rank} \mathbb{S}=\operatorname{dim} S$. The following proposition gives a necessary condition for maximal supersymmetry.

Proposition 11. If a background $(M, g, C, F)$ is maximally supersymmetric, it is flat with respect to the superconnection: the curvature tensor $R^{\mathscr{D}}$, given by

$$
R^{\mathscr{D}}(X, Y)=\mathscr{D}_{[X, Y]}-\left[\mathscr{D}_{X}, \mathscr{D}_{Y}\right]
$$

where $X, Y$ are vector fields, vanishes. The converse holds if $M$ is simply connected.

\footnotetext{
${ }^{4}$ We abuse notation in that on the right-hand side we should have the metrically dual one-form $X^{b}$ and not the vector field, but we trust this ought not be a cause of confusion.
} 
Proof. Clearly from the definition, $R^{\mathscr{D}}$ annihilates Killing spinors. If there are rank $\mathbb{S}=$ $\operatorname{dim} S$ linearly independent Killing spinors, at any point $x \in M$ their values span the fibre $\mathbb{S}_{x}$, thus $R^{\mathscr{D}}$ must annihilate all spinors at $x$, hence $R^{\mathscr{D}}$ vanishes.

Conversely, assume $M$ is simply connected and $\mathscr{D}$ is flat. Any choice of a spinor at any point determines a Killing spinor by parallel transport, and furthermore, parallel transport of a basis at any point determines $\operatorname{dim} S$ linearly independent Killing spinors.

Determining the curvature tensor will allow necessary (and sufficient, in the case of simply-connected backgrounds) conditions for maximal supersymmetry to be found. It is also possible to recover the bosonic supergravity equations by imposing a weaker restriction than the vanishing of the curvature, namely the vanishing of its Clifford trace: $\Gamma^{\nu} R^{\mathscr{D}}{ }_{\mu \nu}=0$. Indeed, after finding $R^{\mathscr{D}}$, our approach will be to calculate its Clifford trace and derive necessary and sufficient conditions for this to vanish, which will simplify the vanishing curvature calculation.

\subsection{Conventions on curvature tensors}

We define the curvature $R^{D} \in \Omega^{2}(M ; \operatorname{End}(E))$ of any connection $D$ on a vector bundle $E$ over $M$ by

$$
R^{D}(X, Y)=D_{[X, Y]}-\left[D_{X}, D_{Y}\right] .
$$

In particular, $R^{\nabla}$ is the Riemann curvature tensor and $R^{\mathscr{D}}$ is the curvature of the superconnection $\mathscr{D}$. The Ricci tensor Ric $\in \odot^{2} T^{*} M$ is

$$
\operatorname{Ric}(X, Y)=\operatorname{tr}\left(Z \mapsto R^{\nabla}(X, Z) Y\right) .
$$

In a local frame ${ }^{5}\left\{\boldsymbol{e}_{\mu}\right\}$, we define the components of the Riemann curvature tensor to be

$$
R^{\nabla}\left(\boldsymbol{e}_{\mu}, \boldsymbol{e}_{\nu}\right) \boldsymbol{e}_{\tau}=R_{\mu \nu}^{\sigma}{ }^{\sigma} e_{\sigma} .
$$

We use the metric to raise and lower indices so that

$$
R_{\mu \nu \sigma \tau}=g_{\sigma \rho} R_{\mu \nu}^{\rho}{ }_{\tau}=g\left(\boldsymbol{e}_{\sigma}, R^{\nabla}\left(\boldsymbol{e}_{\mu}, \boldsymbol{e}_{\nu}\right) e_{\tau}\right)
$$

We denote the corresponding Riemann tensor in $\wedge^{2} \odot^{2} T^{*} M$ with these components by Riem:

$$
\operatorname{Riem}(W, X, Y, Z)=g\left(Y, R^{\nabla}(W, X) Z\right)
$$

The components of the Ricci tensor are then

$$
R_{\mu \nu}=\operatorname{Ric}\left(\boldsymbol{e}_{\mu}, \boldsymbol{e}_{\nu}\right)=R_{\mu \rho}^{\rho}{ }_{\nu}
$$

and the scalar curvature is the trace of the Ricci endomorphism defined by $\operatorname{Ric}(X, Y)=$ $g(X, \operatorname{Ric}(Y))$, or

$$
R=g^{\mu \nu} R_{\mu \nu}
$$

\footnotetext{
${ }^{5}$ Coordinate frame or local orthonormal frame. Recall that in the latter case, $g_{\mu \nu}=\eta_{\mu \nu}$.
} 
Since the Riemann curvature tensor $R^{\nabla}(X, Y)$ takes values in $\mathfrak{s o}(T M)$, it defines an element in the Clifford bundle as in (2.11), so the action on spinors fields $s$ is given by

$$
R^{\nabla}(X, Y) \cdot s=\frac{1}{4} R_{\mu \nu \sigma \tau} X^{\mu} Y^{\nu} \Gamma^{\sigma \tau} s .
$$

We will also use the Weyl tensor

$$
W=\operatorname{Riem}+\frac{R}{2 n(n-1)} g \oplus g+\frac{1}{n-2}\left(\operatorname{Ric}-\frac{R}{n} g\right) \oplus g,
$$

which in components is

$$
\begin{aligned}
W_{\mu \nu \rho \sigma}= & R_{\mu \nu \rho \sigma}+\frac{1}{n-2}\left(R_{\mu \rho} g_{\nu \sigma}+R_{\nu \sigma} g_{\mu \rho}-R_{\mu \sigma} g_{\nu \rho}-R_{\nu \rho} g_{\mu \sigma}\right) \\
& -\frac{R}{(n-1)(n-2)}\left(g_{\mu \rho} g_{\nu \sigma}-g_{\mu \sigma} g_{\nu \rho}\right) .
\end{aligned}
$$

\subsection{Determination of the superconnection curvature}

From the definition of the curvature of the superconnection $R^{\mathscr{D}}$ and the Riemann curvature $R^{\nabla}$, we have

$$
\begin{aligned}
R^{\mathscr{D}}(X, Y) s & =\mathscr{D}_{[X, Y]} s-\left[\mathscr{D}_{X}, \mathscr{D}_{Y}\right] s \\
& =\nabla_{[X, Y]} s-\beta_{[X, Y]} s-\left[\nabla_{X}, \nabla_{Y}\right] s-\left[\beta_{X}, \beta_{Y}\right] s+\left[\nabla_{X}, \beta_{Y}\right] s+\left[\beta_{X}, \nabla_{Y}\right] s \\
& =R^{\nabla}(X, Y) \cdot s-\beta_{[X, Y]} s-\left[\beta_{X}, \beta_{Y}\right] s+\left(\nabla_{X} \beta_{Y}\right) s-\left(\nabla_{Y} \beta_{X}\right) s
\end{aligned}
$$

for all $s \in \Gamma(\mathbb{S})$. Locally, we can write

$$
R_{\mu \nu B}^{\mathscr{D}}{ }^{A}=\frac{1}{4} R_{\mu \nu \sigma \tau} \Gamma^{\sigma \tau} \delta_{B}^{A}-\left[\beta_{\mu}, \beta_{\nu}\right]_{B}^{A}+2 \nabla_{[\mu} \beta_{\nu]}^{A}{ }_{B} .
$$

We'll expand each of the $\beta$ terms in turn. The components $\beta_{\mu}{ }_{B}^{A}$ of $\beta$ are defined by $\left(\beta_{\mu} s\right)^{A}=\beta_{\mu}{ }_{B} s^{B}$. The differential term, straightforwardly, is

$$
\nabla_{[\mu} \beta_{\nu]}{ }_{B}^{A}=-\frac{1}{8} \epsilon_{\alpha \beta \sigma \tau[\mu} \nabla_{\nu]} C^{\alpha \beta} \Gamma^{\sigma \tau} \delta_{B}^{A}+\nabla_{[\mu} C_{\nu] \sigma} \Gamma^{\sigma} \delta_{B}^{A}-\frac{1}{4} \eta_{\sigma[\mu} \nabla_{\nu]} F_{\tau}{ }_{B}^{A} \Gamma^{\sigma \tau}-\frac{1}{2} \nabla_{[\mu} F_{\nu]}{ }_{B}^{A} .
$$

For the commutator $\left[\beta_{\mu}, \beta_{\nu}\right]=2\left(\beta_{[\mu} \beta_{\nu]}\right)$, we first compute

$$
\begin{aligned}
& \beta_{\mu}{ }^{A}{ }_{C} \beta_{\nu}{ }^{C}{ }_{B} \\
&= \frac{1}{64} C^{\alpha \beta} C^{\gamma \delta}\left(\epsilon_{\mu \alpha \beta \sigma \tau} \Gamma^{\sigma \tau}+8 \eta_{\mu \alpha} \Gamma_{\beta}\right)\left(\epsilon_{\nu \gamma \delta \kappa \lambda} \Gamma^{\kappa \lambda}+8 \eta_{\nu \gamma} \Gamma_{\delta}\right) \delta_{B}^{A} \\
&+\frac{1}{16} F_{C}^{\alpha A} F_{B}^{\beta C}\left(\Gamma_{\mu \alpha}-2 \eta_{\mu \alpha}\right)\left(\Gamma_{\nu \beta}-2 \eta_{\nu \beta}\right) \\
&+\frac{1}{32} C^{\alpha \beta} F_{B}^{\gamma A}\left[\left(\epsilon_{\mu \alpha \beta \sigma \tau} \Gamma^{\sigma \tau}+8 \eta_{\mu \alpha} \Gamma_{\beta}\right)\left(\Gamma_{\nu \gamma}-2 \eta_{\nu \gamma}\right)+\left(\Gamma_{\mu \gamma}-2 \eta_{\mu \gamma}\right)\left(\epsilon_{\nu \alpha \beta \sigma \tau} \Gamma^{\sigma \tau}+8 \eta_{\nu \alpha} \Gamma_{\beta}\right)\right] .
\end{aligned}
$$


We will refer to the collections of terms in this expression proportional to $C C, F F$ and $C F$ as $[C C],[F F]$ and $[C F]$ respectively. Expanding $[C C]$ and for now omitting the $\delta_{B}^{A}$, after a long calculation we have

$$
\begin{aligned}
& \frac{1}{64} C^{\alpha \beta} C^{\gamma \delta}\left[\epsilon_{\mu \alpha \beta \sigma \tau} \epsilon_{\nu \gamma \delta \kappa \lambda} \Gamma^{\sigma \tau} \Gamma^{\kappa \lambda}+8 \eta_{\nu \gamma} \epsilon_{\mu \alpha \beta \sigma \tau} \Gamma^{\sigma \tau} \Gamma_{\delta}+8 \eta_{\mu \alpha} \epsilon_{\nu \gamma \delta \kappa \lambda} \Gamma_{\beta} \Gamma^{\kappa \lambda}+64 \eta_{\mu \alpha} \eta_{\nu \gamma} \Gamma_{\beta} \Gamma_{\delta}\right] \\
& =\frac{1}{64} C^{\alpha \beta} C^{\gamma \delta} \epsilon_{\mu \alpha \beta \sigma \tau} \epsilon_{\nu \gamma \delta \kappa \lambda} \Gamma^{\sigma \tau \kappa \lambda}+\frac{1}{8} C^{\alpha \beta}\left(C_{\mu \rho} \epsilon_{\nu \alpha \beta \sigma \tau}+C_{\nu \rho} \epsilon_{\mu \alpha \beta \sigma \tau}\right) \Gamma^{\rho \sigma \tau} \\
& \quad+\frac{5}{4} C_{\mu \alpha} C_{\nu}^{\alpha}-\frac{1}{8} \eta_{\mu \nu} C^{\alpha \beta} C_{\alpha \beta} \\
& \quad+\frac{1}{4}\left(3 C_{\mu \sigma} C_{\nu \tau} \Gamma^{\sigma \tau}-\left(C_{\mu \alpha} C_{\sigma}^{\alpha} \Gamma_{\nu}^{\sigma}-C_{\nu \alpha} C_{\sigma}{ }^{\alpha} \Gamma_{\mu}^{\sigma}\right)-\frac{1}{2} C^{\alpha \beta} C_{\alpha \beta} \Gamma_{\mu \nu}\right) \\
& \quad+\frac{1}{4} C^{\alpha \beta}\left(C_{\mu}^{\gamma} \epsilon_{\nu \alpha \beta \gamma \tau}-C_{\nu}^{\gamma} \epsilon_{\mu \alpha \beta \gamma \tau}\right) \Gamma^{\tau} .
\end{aligned}
$$

We have written the above expression so that terms symmetric in $\mu \nu$ appear first, followed by the skew-symmetric terms. We will do the same with $[F F]$ and $[C F]$. For the former, after another calculation gives

$$
\begin{aligned}
\frac{1}{16} & F_{C}^{\alpha A} F_{B}^{\beta C}\left[\Gamma_{\mu \alpha} \Gamma_{\nu \beta}-2 \eta_{\nu \beta} \Gamma_{\mu \alpha}-2 \eta_{\mu \alpha} \Gamma_{\nu \beta}+4 \eta_{\mu \alpha} \eta_{\nu \beta}\right] \\
= & -\frac{1}{32} \eta_{\mu \nu}\left[F_{\alpha}, F_{\beta}\right]_{B}^{A} \Gamma^{\alpha \beta}+\frac{5}{32}\left\{F_{\mu}, F_{\nu}\right\}_{B}^{A}-\frac{1}{16} \eta_{\mu \nu}\left(F^{\alpha} F_{\alpha}\right)_{B}^{A} \\
& -\frac{1}{32}\left[F_{\alpha}, F_{\beta}\right]_{B}^{A}{ }_{B} \Gamma_{\mu \nu}{ }^{\alpha \beta}-\frac{1}{16}\left(F^{\alpha} F_{\alpha}\right)^{A}{ }_{B} \Gamma_{\mu \nu}+\frac{3}{32}\left[F_{\mu}, F_{\nu}\right]_{B}^{A} \\
& +\frac{1}{16}\left[\left(F_{\nu} F^{\alpha}-2 F^{\alpha} F_{\nu}\right)_{B}^{A}{ }_{B} \Gamma_{\mu \alpha}-\left(2 F_{\mu} F^{\alpha}+F^{\alpha} F_{\mu}\right)_{B}^{A}{ }_{B} \Gamma_{\nu \alpha}\right],
\end{aligned}
$$

where we have separated terms which are explicitly symmetric, skew-symmetric and of indeterminate symmetry in $\mu \nu$. Turning finally to $[C F]$, we have

$$
\begin{aligned}
& \frac{1}{32} C^{\alpha \beta} F_{B}^{\gamma A}\left[\left(\epsilon_{\mu \alpha \beta \sigma \tau} \Gamma^{\sigma \tau}+8 \eta_{\mu \alpha} \Gamma_{\beta}\right)\left(\Gamma_{\nu \gamma}-2 \eta_{\nu \gamma}\right)+\left(\Gamma_{\mu \gamma}-2 \eta_{\mu \gamma}\right)\left(\epsilon_{\nu \alpha \beta \sigma \tau} \Gamma^{\sigma \tau}+8 \eta_{\nu \alpha} \Gamma_{\beta}\right)\right] \\
& =\frac{1}{16} \epsilon_{\alpha \beta \sigma \tau(\mu} C^{\alpha \beta} F_{B}^{\gamma A} \Gamma^{\sigma \tau}{ }_{\nu) \gamma}+\frac{1}{2} F_{\tau}{ }_{B}{ }_{B} C_{\sigma(\mu} \Gamma_{\nu)}{ }^{\sigma \tau}-\frac{1}{4} C^{\alpha \beta} F_{(\mu}{ }_{B}{ }_{B} \epsilon_{\nu) \alpha \beta \sigma \tau} \Gamma^{\sigma \tau}+C_{\sigma(\mu} F_{\nu)}{ }_{B} \Gamma^{\sigma} \\
& +\frac{1}{8} C^{\alpha \beta}\left(-\epsilon_{\mu \nu \alpha \beta \sigma} F_{\tau}{ }^{A}{ }_{B}+\eta_{\sigma[\mu} \epsilon_{\nu] \alpha \beta \gamma \tau} F_{B}^{\gamma A}\right) \Gamma^{\sigma \tau}+\frac{1}{2}\left(C_{\mu \nu} F_{\sigma}+\eta_{\sigma[\mu} C_{\nu] \alpha} F_{B}^{\alpha A}\right) \Gamma^{\sigma} .
\end{aligned}
$$

We now have all of the terms of the commutator $\left[\beta_{\mu}, \beta_{\nu}\right]$. This has two $\Delta$-indices, and after lowering both, it can be separated into symmetric and skew-symmetric parts with respect to these indices. The symmetric part is proportional to $\epsilon$ and the skew-symmetric part takes values in $\mathfrak{s p}(1)$. We must therefore determine the symmetry of the $\Delta$ indices in each term. The following lemma addresses this.

Lemma 12. $\left[F_{\mu}, F_{\nu}\right]_{A B}$ is symmetric in $A B$ and $\left\{F_{\mu}, F_{\nu}\right\}_{A B}$ is skew-symmetric.

Proof. The result follows immediately from the fact that $F$ takes values in $\mathfrak{s p}(1)$. Alternatively, we can show this purely by an exercise in indices: note that $F_{A B}$ is symmetric in $A B$ and consider the product $F_{\mu} F_{\nu}$ with lowered indices:

$$
\begin{aligned}
\left(F_{\mu} F_{\nu}\right)_{A B} & =F_{\mu A C} F_{\nu}{ }^{C}{ }_{B}=\epsilon^{D C} F_{\mu A C} F_{\nu D B}=-\epsilon^{C D} F_{\nu B D} F_{\mu C A} \\
& =-F_{\nu B D} F_{\mu}{ }^{D}=-\left(F_{\nu} F_{\mu}\right)_{B A} ;
\end{aligned}
$$


we thus have

$$
\left(F_{(\mu} F_{\nu)}\right)_{A B}=\left(F_{(\nu} F_{\mu)}\right)_{A B}=-\left(F_{(\mu} F_{\nu)}\right)_{B A}, \quad\left(F_{[\mu} F_{\nu]}\right)_{A B}=-\left(F_{[\nu} F_{\mu]}\right)_{A B}=\left(F_{[\mu} F_{\nu]}\right)_{B A},
$$

hence the result.

We now evaluate the commutator. Since $\left[\beta_{\mu}, \beta_{\nu}\right]=2\left(\beta_{[\mu} \beta_{\nu]}\right)$, only the $\mu \nu$-skewsymmetric terms of $[C C],[C F]$ and $[F F]$ contribute. Organising the terms of the commutator by their $\Delta$-symmetry but suppressing those indices for convenience, the commutator $\left[\beta_{\mu}, \beta_{\nu}\right]$ is

$$
\begin{aligned}
& \frac{1}{4}\left[\left(6 C_{\mu \sigma} C_{\nu \tau}+4 \eta_{\sigma[\mu} C_{\nu] \alpha} C_{\tau}{ }^{\alpha}-C^{\alpha \beta} C_{\alpha \beta} \eta_{\mu \sigma} \eta_{\nu \tau}\right) \epsilon+\frac{1}{2}\left(\eta_{\sigma[\mu}\left\{F_{\nu]}, F_{\tau}\right\}-\left(F^{\alpha} F_{\alpha}\right) \eta_{\mu \sigma} \eta_{\nu \tau}\right)\right] \Gamma^{\sigma \tau} \\
& +\epsilon_{\alpha \beta \gamma \sigma[\mu} C^{\alpha \beta} C_{\nu]}^{\gamma} \epsilon \Gamma^{\sigma}+\frac{1}{4}\left[\eta_{\sigma[\mu}\left[F_{\nu]}, F_{\tau}\right]-\left(\epsilon_{\mu \nu \alpha \beta \sigma} C^{\alpha \beta} F_{\tau}-\eta_{\sigma[\mu} \epsilon_{\nu] \alpha \beta \gamma \tau} C^{\alpha \beta} F^{\gamma}\right)\right] \Gamma^{\sigma \tau} \\
& +\left[-\frac{1}{16} \epsilon_{\mu \nu \alpha \beta \sigma}\left[F^{\alpha}, F^{\beta}\right]+C_{\mu \nu} F_{\sigma}+\eta_{\sigma[\mu} C_{\nu] \alpha} F^{\alpha}\right] \Gamma^{\sigma}+\frac{3}{16}\left[F_{\mu}, F_{\nu}\right] .
\end{aligned}
$$

An explicit expression for the curvature of the superconnection in terms of $C$ and $F$ can now finally be found by substituting equations (4.19) and (4.26) into equation (4.18). For the sake of readability, we will first define some new notation. It will be useful to denote the components of $R^{\mathscr{D}}$ as follows:

$$
R_{\mu \nu A B}^{\mathscr{D}}=\frac{1}{2} L_{\mu \nu \sigma \tau A B} \Gamma^{\sigma \tau}+M_{\mu \nu \sigma A B} \Gamma^{\sigma}+N_{\mu \nu A B},
$$

where

$$
\begin{array}{rlrlrl}
L & =L^{\wedge} \epsilon_{A B}+L_{A B}^{\odot} & L^{\wedge} & \in \Omega^{2}\left(M ; \wedge^{2} T M\right) & L^{\odot} \in \Omega^{2}\left(M ; \wedge^{2} T M \otimes \mathfrak{s p}(1)\right) \\
M & =M^{\wedge} \epsilon_{A B}+M_{A B}^{\odot} & M^{\wedge} & \in \Omega^{2}\left(M ; \wedge^{1} T M\right) & & M^{\odot} \in \Omega^{2}\left(M ; \wedge^{1} T M \otimes \mathfrak{s p}(1)\right) \\
N & =N^{\wedge} \epsilon_{A B}+N_{A B}^{\odot} & N^{\wedge} & \in \Omega^{2}(M) & & N^{\odot} \in \Omega^{2}(M ; \mathfrak{s p}(1)) .
\end{array}
$$

Said another way, $L_{\mu \nu \sigma \tau A B}, M_{\mu \nu \sigma A B}$ and $N_{\mu \nu A B}$ are skew-symmetric in $\mu \nu$, and $L_{\mu \nu \sigma \tau A B}$ is also skew-symmetric in $\sigma \tau ; L^{\wedge}$ and $L^{\odot}$ are respectively the skew-symmetric and symmetric parts of $L$ (with respect to $\Delta$-indices).

Since it is skew-symmetric, the anticommutator $\left\{F_{\mu}, F_{\nu}\right\}$ can be written in terms of its trace: $\left\{F_{\mu}, F_{\nu}\right\}_{A B}=\frac{1}{2}\left\{F_{\mu}, F_{\nu}\right\}_{C}^{C} \epsilon_{A B}$. Let us define $F_{\mu} \cdot F_{\nu}=F_{\mu}{ }^{A B} F_{\nu A B}=-\left(F_{\mu} F_{\nu}\right)_{A}^{A}$ and $F^{2}=F^{\alpha} \cdot F_{\alpha}$. Note that $F_{\mu} \cdot F_{\nu}=F_{\nu} \cdot F_{\mu}$, so

$$
\left\{F_{\mu}, F_{\nu}\right\}_{A B}=-\left(F_{\mu} \cdot F_{\nu}\right) \epsilon_{A B} \quad \text { and } \quad\left(F_{\alpha} F^{\alpha}\right)_{A B}=-\frac{1}{2} F^{2} \epsilon_{A B}
$$


We thus have

$$
\begin{aligned}
L^{\wedge}{ }_{\mu \nu \sigma \tau}= & \frac{1}{2}\left[R_{\mu \nu \sigma \tau}-\left(6 C_{\mu[\sigma} C_{|\nu| \tau]}+2 \eta_{\mu[\sigma} C_{|\nu \alpha|} C_{\tau]}{ }^{\alpha}-2 \eta_{\nu[\sigma} C_{|\mu \alpha|} C_{\tau]}{ }^{\alpha}-C^{\alpha \beta} C_{\alpha \beta} \eta_{\mu[\sigma} \eta_{|\nu| \tau]}\right)\right. \\
& \left.-\epsilon_{\alpha \beta \sigma \tau[\mu} \nabla_{\nu]} C^{\alpha \beta}+\frac{1}{4}\left(\eta_{\mu[\sigma}\left(F_{|\nu|} \cdot F_{\tau]}\right)-\eta_{\nu[\sigma}\left(F_{|\mu|} \cdot F_{\tau]}\right)-F^{2} \eta_{\mu[\sigma} \eta_{|\nu| \tau]}\right)\right] \\
M_{\mu \nu \sigma}^{\wedge}= & -\epsilon_{\alpha \beta \gamma \sigma[\mu} C^{\alpha \beta} C_{\nu]}^{\gamma}+2 \nabla_{[\mu} C_{\nu] \sigma} \\
N_{\mu \nu}^{\wedge}= & 0 \\
L^{\odot}{ }_{\mu \nu \sigma \tau}= & -\frac{1}{4}\left(\eta_{\mu[\sigma}\left[F_{|\nu|}, F_{\tau]}\right]-\eta_{\nu[\sigma}\left[F_{|\mu|}, F_{\tau]}\right]\right)+\frac{1}{2} \epsilon_{\mu \nu \alpha \beta[\sigma} C^{\alpha \beta} F_{\tau]} \\
& -\frac{1}{4}\left(\eta_{\mu[\sigma} \epsilon_{\tau] \nu \alpha \beta \gamma} C^{\alpha \beta} F^{\gamma}-\eta_{\nu[\sigma} \epsilon_{\tau] \mu \alpha \beta \gamma} C^{\alpha \beta} F^{\gamma}\right)-\frac{1}{2}\left(\eta_{\mu[\sigma} \nabla_{|\nu|} F_{\tau]}-\eta_{\nu[\sigma} \nabla_{|\mu|} F_{\tau]}\right) \\
M^{\odot}{ }_{\mu \nu \sigma}= & \frac{1}{16} \epsilon_{\mu \nu \alpha \beta \sigma}\left[F^{\alpha}, F^{\beta}\right]-C_{\mu \nu} F_{\sigma}-\eta_{\sigma[\mu} C_{\nu] \alpha} F^{\alpha} \\
N^{\odot}{ }_{\mu \nu}= & -\frac{3}{16}\left[F_{\mu}, F_{\nu}\right]-\nabla_{[\mu} F_{\nu]} .
\end{aligned}
$$

This explicitly gives the superconnection curvature $R^{\mathscr{D}}$ in terms of the background fields. We now go on to show how equations of motion and maximal supersymmetry conditions can be extracted from the curvature.

\subsection{Clifford trace of superconnection curvature}

In this section we seek to compute necessary and sufficient conditions for the Clifford trace of the curvature $\Gamma^{\nu} R^{\mathscr{D}}{ }_{\mu \nu}$ to vanish identically as a one-form with values in $\operatorname{End}(\mathbb{S})$. To this end we will make use of some of the identities from appendix A.

Theorem 13. Let $(M, g, C, F)$ be a 5-dimensional background with superconnection $\mathscr{D}$ given by equation (4.3). The Clifford trace of the curvature $\Gamma^{\nu} R^{\mathscr{D}}{ }_{\mu \nu}$ vanishes if and only if the following equations hold:

$$
\begin{aligned}
& \nabla^{\alpha} C_{\alpha \mu}=\frac{1}{2} \epsilon_{\mu \alpha \beta \gamma \delta} C^{\alpha \beta} C^{\gamma \delta} \\
& C_{\mu \alpha} F^{\alpha}=0 \\
& \nabla_{[\sigma} C_{\mu \nu]}=0 \\
& R_{\mu \nu}+\left(6 C_{\mu \alpha} C_{\nu}^{\alpha}-\eta_{\mu \nu} C^{\alpha \beta} C_{\alpha \beta}\right)-\frac{3}{8}\left(\left(F_{\mu} \cdot F_{\nu}\right)-\eta_{\mu \nu} F^{2}\right)=0 \\
& \nabla_{\mu} F_{\nu}=-\frac{1}{2} \epsilon_{\mu \nu \alpha \beta \gamma} C^{\alpha \beta} F^{\gamma} \\
& {\left[F_{\mu}, F_{\nu}\right]=0 .}
\end{aligned}
$$

Equation (4.38) is simply $d C=0$, or $C$ is closed, consistent with $C$ being the field strength tensor of a one-form potential as in supergravity. Equation (4.37) is simply $\iota_{F_{A B}} C=0$. Equation (4.36) is the Maxwell-like supergravity bosonic equation of motion, while if we set $F=0$, equation (4.39) becomes the Einstein-like equation. Equation (4.40) provides a third equation of motion wherever $F \neq 0$. Writing $\Delta$-indices, we also learn 
that each $F_{A B}$ is a Killing vector field and (from equation (4.41)) that the $F_{\mu}$ commute everywhere under the $\mathfrak{s p}(1)$ commutator.

Proof. Using $L, M, N$ as defined above,

$$
\begin{aligned}
\Gamma^{\nu} R_{\mu \nu}^{\mathscr{D}} & =\frac{1}{2} L_{\mu \nu \sigma \tau}\left(\Gamma^{\nu \sigma \tau}+2 \eta^{\nu[\sigma} \Gamma^{\tau]}\right)+M_{\mu \nu \sigma}\left(\Gamma^{\nu \sigma}+\eta^{\nu \sigma}\right)+N_{\mu \nu} \Gamma^{\nu}, \\
& =\left(-\frac{1}{4} \epsilon_{\alpha \beta \gamma \sigma \tau} L_{\mu}{ }^{\alpha \beta \gamma}+M_{\mu \sigma \tau}\right) \Gamma^{\sigma \tau}+\left(L_{\mu}^{\alpha} \alpha \sigma+N_{\mu \sigma}\right) \Gamma^{\sigma}+M_{\mu}^{\alpha} \alpha
\end{aligned}
$$

For the first term we have

$$
\begin{aligned}
L^{\wedge}{ }_{\mu[\alpha \beta \gamma]} & =3 C_{\mu[\alpha} C_{\beta \gamma]}+\frac{1}{4}\left(\epsilon_{\kappa \lambda \alpha \beta \gamma} \nabla_{\mu} C^{\kappa \lambda}-\epsilon_{\mu \kappa \lambda[\alpha \beta} \nabla_{\gamma]} C^{\kappa \lambda}\right), \\
L^{\odot}{ }_{\mu[\alpha \beta \gamma]} & =\frac{1}{4} \eta_{\mu[\alpha}\left[F_{\beta}, F_{\gamma]}\right]+\frac{1}{2} \epsilon_{\mu \kappa \lambda[\alpha \beta} C^{\kappa \lambda} F_{\gamma]}-\frac{1}{4} \eta_{\mu[\alpha} \epsilon_{\beta \gamma] \kappa \lambda \rho} C^{\kappa \lambda} F^{\rho}+\frac{1}{2} \eta_{\mu[\alpha} \nabla_{\beta} F_{\gamma]},
\end{aligned}
$$

thus

$$
\begin{aligned}
-\frac{1}{4} \epsilon_{\alpha \beta \gamma \sigma \tau} L^{\wedge}{ }_{\mu}^{\alpha \beta \gamma}= & -\frac{3}{4} \epsilon_{\alpha \beta \gamma \sigma \tau} C_{\mu}{ }^{\alpha} C^{\beta \gamma}-\frac{1}{2} \nabla_{\mu} C_{\sigma \tau}-\frac{1}{2} \eta_{\mu[\sigma} \nabla^{\gamma} C_{|\gamma| \tau]} \\
-\frac{1}{4} \epsilon_{\alpha \beta \gamma \sigma \tau} L^{\odot}{ }_{\mu}^{\alpha \beta \gamma}= & -\frac{1}{16} \epsilon_{\mu \alpha \beta \sigma \tau}\left[F^{\alpha}, F^{\beta}\right]-\frac{1}{4} C_{\sigma \tau} F_{\mu}+\frac{1}{2} C_{\mu[\sigma} F_{\tau]}-\eta_{\mu[\sigma} C_{\tau] \gamma} F^{\gamma} \\
& -\frac{1}{8} \epsilon_{\mu \alpha \beta \sigma \tau} \nabla^{\alpha} F^{\beta} .
\end{aligned}
$$

The trace in the second term is

$$
\begin{aligned}
L_{\mu \alpha \alpha \sigma}^{\wedge}{ }_{\mu}^{\alpha} & =\frac{1}{2}\left[R_{\mu \sigma}+\left(6 C_{\mu \alpha} C_{\sigma}{ }^{\alpha}-\eta_{\mu \sigma} C^{\alpha \beta} C_{\alpha \beta}\right)-\frac{3}{8}\left(\left(F_{\mu} \cdot F_{\sigma}\right)-\eta_{\mu \sigma} F^{2}\right)+\frac{1}{2} \epsilon_{\mu \sigma \alpha \beta \gamma} \nabla^{\alpha} C^{\beta \gamma}\right], \\
L^{\odot}{ }_{\mu \alpha \sigma}^{\alpha} & =\frac{3}{8}\left[F_{\mu}, F_{\sigma}\right]-\frac{1}{8} \epsilon_{\mu \sigma \alpha \beta \gamma} C^{\alpha \beta} F^{\gamma}+\frac{1}{4}\left(\eta_{\mu \sigma} \nabla_{\alpha} F^{\alpha}+3 \nabla_{\mu} F_{\sigma}\right) .
\end{aligned}
$$

We also need

$$
\begin{aligned}
M_{\mu[\sigma \tau]}^{\wedge} & =-\frac{1}{2} \epsilon_{\mu \alpha \beta \gamma[\sigma} C_{\tau]}^{\alpha} C^{\beta \gamma}+\frac{1}{2} \epsilon_{\alpha \beta \gamma \sigma \tau} C_{\mu}^{\alpha} C^{\beta \gamma}+\nabla_{\mu} C_{\sigma \tau}+\nabla_{[\sigma} C_{\tau] \mu}, \\
M^{\wedge}{ }_{\mu \alpha}^{\alpha} & =-\frac{1}{2} \epsilon_{\mu \alpha \beta \gamma \delta} C^{\alpha \beta} C^{\gamma \delta}+\nabla^{\alpha} C_{\alpha \mu}, \\
M^{\odot}{ }_{\mu[\sigma \tau]} & =\frac{1}{16} \epsilon_{\mu \sigma \tau \alpha \beta}\left[F^{\alpha}, F^{\beta}\right]-C_{\mu[\sigma} F_{\tau]}+\frac{1}{2} \eta_{\mu[\sigma} C_{\tau] \alpha} F^{\alpha}, \\
M^{\odot}{ }_{\mu \alpha}^{\alpha} & =C_{\mu \alpha} F^{\alpha} .
\end{aligned}
$$

It follows immediately from equation (4.42) that $\Gamma^{\nu} R^{\mathscr{D}}{ }_{\mu \nu}$ vanishes if and only if

$$
\begin{aligned}
& -\frac{1}{4} \epsilon_{\alpha \beta \gamma \sigma \tau} L_{\mu}^{\wedge}{ }^{\alpha \beta \gamma}+M^{\wedge}{ }_{\mu[\sigma \tau]}=0, \quad-\frac{1}{4} \epsilon_{\alpha \beta \gamma \sigma \tau} L^{\odot}{ }_{\mu}^{\alpha \beta \gamma}+M^{\odot}{ }_{\mu[\sigma \tau]}=0, \\
& L_{\mu \alpha \sigma}^{\wedge}{ }_{\mu \alpha}^{\alpha}=0, \quad L^{\odot}{ }_{\mu \alpha \sigma}^{\alpha}+N^{\odot}{ }_{\mu \sigma}=0, \\
& M_{\mu \alpha}^{\wedge \alpha}=0, \quad M_{\mu \alpha}^{\odot}{ }_{\mu}^{\alpha}=0 .
\end{aligned}
$$


Expanding each of these out, we obtain equations (4.36) and (4.37) as well as

$$
\begin{aligned}
-\frac{1}{4} \epsilon_{\alpha \beta \gamma \sigma \tau} C_{\mu}{ }^{\alpha} C^{\beta \gamma}-\frac{1}{2} \epsilon_{\mu \alpha \beta \gamma[\sigma} C_{\tau]}{ }^{\alpha} C^{\beta \gamma}+\frac{3}{2} \nabla_{[\mu} C_{\sigma \tau]}-\frac{1}{2} \eta_{\mu[\sigma} \nabla^{\gamma} C_{|\gamma| \tau]} & =0 \\
R_{\mu \sigma}+\left(6 C_{\mu \alpha} C_{\sigma}{ }^{\alpha}-\eta_{\mu \sigma} C^{\alpha \beta} C_{\alpha \beta}\right)-\frac{3}{8}\left(\left(F_{\mu} \cdot F_{\sigma}\right)-\eta_{\mu \sigma} F^{2}\right)+\frac{1}{2} \epsilon_{\mu \sigma \alpha \beta \gamma} \nabla^{\alpha} C^{\beta \gamma} & =0 \\
-\frac{3}{4} C_{[\sigma \tau} F_{\mu]}-\frac{1}{2} \eta_{\mu[\sigma} C_{\tau] \gamma} F^{\gamma}-\frac{1}{8} \epsilon_{\mu \alpha \beta \sigma \tau} \nabla^{\alpha} F^{\beta} & =0 \\
\frac{3}{16}\left[F_{\mu}, F_{\sigma}\right]-\frac{1}{4} \nabla_{[\mu} F_{\sigma]}-\frac{1}{8} \epsilon_{\mu \sigma \alpha \beta \gamma} C^{\alpha \beta} F^{\gamma}+\frac{1}{4}\left(\eta_{\mu \sigma} \nabla_{\alpha} F^{\alpha}+3 \nabla_{(\mu} F_{\sigma)}\right) & =0
\end{aligned}
$$

We'll tackle these equations by splitting each of them into irreducible $\mathfrak{s o}(V)$-module components. Taking equation (4.54) first, its full skew-symmetrisation is

$$
\frac{1}{4} \epsilon_{\alpha \beta \gamma[\sigma \tau} C_{\mu]}^{\alpha} C^{\beta \gamma}+\frac{3}{2} \nabla_{[\mu} C_{\sigma \tau]}=0
$$

The first term vanishes identically by (A.4) from appendix A, so this is equivalent to equation (4.38). The trace of (4.54) is

$$
\frac{1}{2} \epsilon_{\tau \alpha \beta \gamma \delta} C^{\alpha \beta} C^{\gamma \delta}-\nabla^{\alpha} C_{\alpha \tau}=0,
$$

which gives us equation (4.36) again. What remains of (4.54) after subtracting off the skew and trace parts is

$$
-\frac{1}{4} \epsilon_{\alpha \beta \gamma \sigma \tau} C_{\mu}^{\alpha} C^{\beta \gamma}-\frac{1}{2} \epsilon_{\mu \alpha \beta \gamma[\sigma} C_{\tau]}^{\alpha} C^{\beta \gamma}-\frac{1}{4} \eta_{\mu[\sigma} \epsilon_{\tau] \alpha \beta \gamma \delta} C^{\alpha \beta} C^{\gamma \delta}=0
$$

which using the tensor identities (A.4) and (A.5) from appendix A is trivial. The skewsymmetric part of equation (4.55) gives equation (4.38) again. The symmetric part is equation (4.39). The totally skew-symmetric part of equation (4.56) is

$$
-\frac{3}{4} C_{[\sigma \tau} F_{\mu]}-\frac{1}{8} \epsilon_{\mu \sigma \tau \alpha \beta} \nabla^{\alpha} F^{\beta}=0 .
$$

The trace gives equation (4.37) again. The remaining part vanishes identically. The skewsymmetric part of equation (4.57) is

$$
\frac{3}{16}\left[F_{\mu}, F_{\sigma}\right]-\frac{1}{4} \nabla_{[\mu} F_{\sigma]}-\frac{1}{8} \epsilon_{\mu \sigma \alpha \beta \gamma} C^{\alpha \beta} F^{\gamma}=0,
$$

and the symmetric part is

$$
\frac{1}{4}\left(\eta_{\mu \sigma} \nabla_{\alpha} F^{\alpha}+3 \nabla_{(\mu} F_{\sigma)}\right)=0
$$

Tracing this equation gives $\nabla_{\alpha} F^{\alpha}=0$, which can be back-substituted to give $\nabla_{(\mu} F_{\sigma)}=0$. Thus contracting equation (4.61) with $\epsilon^{\kappa \lambda \mu \sigma \tau}$ yields equation (4.40). Substituting that equation into equation (4.62) finally produces equation (4.41). 


\subsection{Flatness of the superconnection and maximal supersymmetry}

We now turn to finding conditions on $(M, g, C, F)$ equivalent to the vanishing of the curvature of the superconnection, which by Proposition 11 is necessary for the background to be maximally supersymmetric. We will prove the following theorem.

Theorem 14. Let $(M, g, C, F)$ be a 5-dimensional background with superconnection $\mathscr{D}$ given by equation (4.3). If it is maximally supersymmetric then $C=0$ or $F=0$, and

1. If $C=0$ and $F=0$ then the Riemann curvature vanishes.

2. If $C=0$ and $F \neq 0$ then $F=\varphi \otimes r$ for some parallel one-form $\varphi$ and some $r \in \mathfrak{s p}(1)$, and the Riemann curvature is given by

$$
R_{\mu \nu \sigma \tau}=\varphi^{2} \eta_{\mu[\sigma} \eta_{|\nu| \tau]}-\left(\eta_{\mu[\sigma} \varphi_{|\nu|} \varphi_{\tau]}-\eta_{\nu[\sigma} \varphi_{|\mu|} \varphi_{\tau]}\right) .
$$

3. If $F=0$ and $C \neq 0$ then $C$ is a closed 2-form such that

$$
\nabla_{\sigma} C_{\mu \nu}=\frac{1}{2} \eta_{\sigma[\mu} \nabla^{\alpha} C_{|\alpha| \nu]}=\frac{1}{4} \eta_{\sigma[\mu} \epsilon_{\nu] \alpha \beta \gamma \delta} C^{\alpha \beta} C^{\gamma \delta},
$$

and the Riemann curvature is given by

$$
R_{\mu \nu \sigma \tau}=2 C_{\mu \nu} C_{\sigma \tau}+2 C_{\mu[\sigma} C_{|\nu| \tau]}+2 \eta_{\mu[\sigma} C_{|\nu \alpha|} C_{\tau]}^{\alpha}-2 \eta_{\nu[\sigma} C_{|\mu \alpha|} C_{\tau]}^{\alpha}-C^{\alpha \beta} C_{\alpha \beta} \eta_{\mu[\sigma} \eta_{|\nu| \tau]} .
$$

Proof. The curvature $R^{\mathscr{D}}{ }_{\mu \nu}$ vanishes if and only if each of the components, given by equations (4.30)-(4.35), vanishes separately. We first simplify the problem by noting that if $R^{\mathscr{D}}$ vanishes, in particular its Clifford trace vanishes, so we may use the conditions derived at the end of the previous subsection. We will also need some of the identities of appendix A. Indeed, using $\left[F_{\mu}, F_{\nu}\right]=0$ and $C_{\mu \alpha} F^{\alpha}=0$, the vanishing of $M^{\odot}$ and $N^{\odot}$ gives

$$
\nabla_{[\mu} F_{\nu]}=0 \quad \text { and } \quad C_{\mu \nu} F_{\sigma}=0,
$$

so in particular, either $F=0$ or $C=0$ and since we already have $\nabla_{(\mu} F_{\nu)}=0$ from the zero Clifford trace equations, $F$ is parallel. Now $L^{\odot}=0$ exactly. Using $d C=0$ and equation (A.4), the vanishing of $M^{\wedge}$ is equivalent to

$$
\nabla_{\sigma} C_{\mu \nu}=\frac{1}{2} \epsilon_{\alpha \beta \gamma \mu \nu} C^{\alpha \beta} C_{\sigma}^{\gamma},
$$

and then using equations (A.5) and (4.36) (which is in fact the trace of equation (4.68)) yields equation (4.65). Now using equation (4.65), $L^{\wedge}=0$ if and only if

$$
\begin{aligned}
R_{\mu \nu \sigma \tau}= & 2 C_{\mu \nu} C_{\sigma \tau}+2 C_{\mu[\sigma} C_{|\nu| \tau]}+2 \eta_{\mu[\sigma} C_{|\nu \alpha|} C_{\tau]}^{\alpha}-2 \eta_{\nu[\sigma} C_{|\mu \alpha|} C_{\tau]}^{\alpha}-C^{\alpha \beta} C_{\alpha \beta} \eta_{\mu[\sigma} \eta_{|\nu| \tau]} \\
& -\frac{1}{4}\left(\eta_{\mu[\sigma}\left(F_{|\nu|} \cdot F_{\tau]}\right)-\eta_{\nu[\sigma}\left(F_{|\mu|} \cdot F_{\tau]}\right)-F^{2} \eta_{\mu[\sigma} \eta_{|\nu| \tau]}\right)
\end{aligned}
$$

which we note can be traced to give equation (4.39). For the $F=0$ case, we get equation (4.66). Finally, for the $C=0$ case, since both $\nabla F=0$ and $\left[F_{\mu}, F_{\nu}\right]=0, F=\varphi \otimes r$, for some one-form $\varphi$ and some fixed $r \in \mathfrak{s p}(1)$. In components, $F_{\mu}^{A B}=\varphi_{\mu} r^{A B}$, so $F_{\mu} \cdot F_{\nu}=\varphi_{\mu} \varphi_{\nu} r^{A B} r_{A B}$. We have $r^{A B} r_{A B}=-\operatorname{tr}(r r)$, where this trace is taken in the vector representation of $\mathfrak{s p}(1) \cong \mathfrak{s u}(2)$ on $\mathbb{C}^{2}$, and is therefore negative-definite. We can assume without loss of generality that $r \neq 0$ (by choosing $\varphi=0$ if $F=0$ ), and then by rescaling $r$ and $\varphi$ we can also assume that $r^{A B} r_{A B}=-\operatorname{tr}(r r)=4$, yielding equation (4.64). 


\section{$5 \quad$ The Killing superalgebra}

In this section we define and prove the existence of the supersymmetry algebra of a supersymmetric background. To do so, we will need the notion of the spinorial derivative, for which we will give a definition and state some properties. We will also need to upgrade our definitions of spinor bilinears from section 2.1 to bilinears of spinor fields and derive some of their differential properties.

\subsection{The spinorial Lie derivative}

Throughout, let $(M, g)$ be a spin manifold with an associated spinor bundle $\mathbb{S}$.

Recall that for any vector field $X, \nabla X$ defines an endomorphism on the $C^{\infty}(M)$ module of vector fields $\mathfrak{X}(M)$ by $Y \mapsto \nabla_{Y} X ; \nabla X$ is a section of $\operatorname{End}(T M)$ with components $(\nabla X)^{\mu}{ }_{\nu}=\nabla_{\nu} X^{\mu}$. Furthermore, $\nabla X$ is actually a section of $\mathfrak{s o}(T M)$ if and only if $X$ is a Killing vector field (this follows directly from the definition), in which case $\nabla X$ has the action on spinors $(\nabla X) s=-\frac{1}{4} \nabla_{\mu} X_{\nu} \Gamma^{\mu \nu} s$. This allows us to make the following definition.

Definition 15 ([17, 18]). The spinorial Lie derivative of a spinor field $s$ along the Killing vector field $X$ is given by

$$
\mathscr{L}_{X} s=\nabla_{X} s-(\nabla X) s .
$$

Locally, we have

$$
\mathscr{L}_{X} s=\nabla_{X} s+\frac{1}{4} \nabla_{\mu} X_{\nu} \Gamma^{\mu \nu} s .
$$

This obeys the Leibniz rule: for a smooth function $f$ and a spinor field $s$,

$$
\begin{aligned}
\mathscr{L}_{X}(f s) & =\nabla_{X}(f s)-(\nabla X)(f s) \\
& =X(f) s+f \nabla_{X} s-f((\nabla X) s) \\
& =X(f) s+f \mathscr{L}_{X} s .
\end{aligned}
$$

We will not prove the following lemma; we refer the reader to $[17,18]$.

Lemma 16. The spinorial Lie derivative obeys the following properties:

1. Representation of the Lie algebra of vector fields on spinor fields:

$$
\mathscr{L}_{X} \mathscr{L}_{Y} s=\mathscr{L}_{\mathscr{L}_{X} Y} s+\mathscr{L}_{Y} \mathscr{L}_{X} s
$$

for all Killing vectors $X, Y$ and all $s \in \Gamma(\mathbb{S})$,

2. Leibniz rule with respect to the Clifford action:

$$
\mathscr{L}_{X}(\Phi \cdot s)=\left(\mathscr{L}_{X} \Phi\right) \cdot s+\Phi \cdot\left(\mathscr{L}_{X} s\right)
$$

for all Killing vectors $X$ and all $\Phi \in \Omega^{\bullet}(M), s \in \Gamma(\mathbb{S})$, 
3. Compatibility with the Levi-Civita connection:

$$
\mathscr{L}_{X} \nabla_{Y} s=\nabla_{\mathscr{L}_{X} Y} s+\nabla_{Y} \mathscr{L}_{X} s
$$

for all Killing vectors $X$ and all $Y \in \mathfrak{X}(M), s \in \Gamma(\mathbb{S})$.

We have an additional result for backgrounds.

Lemma 17. If $(M, g, C, F)$ is a 5-dimensional background with superconnection $\mathscr{D}$,

$$
\mathscr{L}_{X} \mathscr{D}_{Y} s=\mathscr{D}_{\mathscr{L}_{X} Y} s+\mathscr{D}_{Y} \mathscr{L}_{X} s-\left(\mathscr{L}_{X} \beta\right)_{Y} s
$$

for all Killing vectors $X$ and all $Y \in \mathfrak{X}(M), s \in \Gamma(\mathbb{S})$.

Proof. Using Lemma 16 and the definition of $\mathscr{D}$,

$$
\begin{aligned}
\mathscr{L}_{X} \mathscr{D}_{Y} s & =\mathscr{L}_{X} \nabla_{Y} s-\mathscr{L}_{X}\left(\beta_{Y} s\right) \\
& =\nabla \mathscr{L}_{X} s+\nabla_{Y} \mathscr{L}_{X} s-\left(\mathscr{L}_{X} \beta\right)_{Y} s-\beta_{\mathscr{L}_{X} Y} s-\beta_{Y} \mathscr{L}_{X} s \\
& =\mathscr{D}_{\mathscr{L}_{X} Y} s+\mathscr{D}_{Y} \mathscr{L}_{X} s-\left(\mathscr{L}_{X} \beta\right)_{Y} s
\end{aligned}
$$

hence the result.

\subsection{Spinor field bilinears}

We now "geometrise" our definitions of the spinor bilinears equation (2.19). For $s \in \Gamma(\mathbb{S})$, we define $\mu_{s} \in C^{\infty}(M), \kappa_{s} \in \mathfrak{X}(M)$ and $\omega_{s}^{A B} \in \Omega^{2}(M ; \mathbb{C})$ as follows:

$$
\mu_{s}=\langle s, s\rangle, \quad g\left(\kappa_{s}, X\right)=\langle s, X \cdot s\rangle, \quad \omega_{s}^{A B}(X, Y)=\frac{1}{2} \bar{s}^{A}[X, Y] \cdot s^{B},
$$

for all $X, Y \in \mathfrak{X}(M)$. It is then useful to define the (linear) Dirac current map $\kappa: \odot^{2} \Gamma(\mathbb{S}) \rightarrow$ $\mathfrak{X}(M)$ by

$$
g\left(\kappa\left(s_{1}, s_{2}\right), X\right)=\left\langle s_{1}, X \cdot s_{2}\right\rangle
$$

and we note that clearly $\kappa_{s}=\kappa(s, s)$.

Lemma 18. The Dirac current map is equivariant under the action of Killing vector fields via $\mathscr{L}$;

$$
\mathscr{L}_{X} \kappa(s, s)=2 \kappa\left(\mathscr{L}_{X} s, s\right)
$$

for all spinor fields $s \in \Gamma(\mathbb{S})$ and Killing vector fields $X$.

Proof. First, note that since $\kappa$ is symmetric in its arguments, it is sufficient to prove the result on the diagonal since the full result then follows by a polarisation identity. Hence, $X$ be a Killing vector field, $Y$ a vector field and $s$ a spinor field. Then locally,

$$
\begin{aligned}
Y \cdot((\nabla X) s) & =-\frac{1}{4} Y^{\lambda} \nabla_{\mu} X_{\nu} \Gamma_{\lambda} \Gamma^{\mu \nu} s \\
& =-\frac{1}{4} Y_{\lambda} \nabla_{\mu} X_{\nu} \Gamma^{\lambda \mu \nu} s-\frac{1}{2} Y^{\mu} \nabla_{\mu} X_{\nu} \Gamma^{\nu} s \\
& =-\frac{1}{8} \epsilon^{\lambda \mu \nu \sigma \tau} Y_{\lambda} \nabla_{\mu} X_{\nu} \Gamma_{\sigma \tau} \cdot s-\frac{1}{2}\left(\nabla_{Y} X\right) \cdot s
\end{aligned}
$$


and so, since $\left\langle s, \Gamma_{\sigma \tau} s\right\rangle=0$,

$$
\langle s, Y \cdot(\nabla X) s\rangle=-\frac{1}{2}\left\langle s,\left(\nabla_{Y} X\right) \cdot s\right\rangle .
$$

Using this and the definition of the Dirac current, we have

$$
\begin{aligned}
g\left(\mathscr{L}_{X} \kappa(s, s), Y\right) & =X(g(\kappa(s, s), Y))-g\left(\kappa(s, s), \mathscr{L}_{X} Y\right) \\
& =g\left(\nabla_{X} \kappa(s, s), Y\right)+g\left(\kappa(s, s), \nabla_{X} Y\right)-g(\kappa(s, s),[X, Y]) \\
& =2 g\left(\kappa\left(s, \nabla_{X} s\right), Y\right)+g\left(\kappa(s, s), \nabla_{Y} X\right) \\
& =\left\langle s, 2 Y \cdot\left(\nabla_{X} s\right)+\left(\nabla_{Y} X\right) \cdot s\right\rangle \\
& =\left\langle s, 2 Y \cdot\left(\nabla_{X} s-(\nabla X) s\right)\right\rangle \\
& =2\left\langle s, Y \cdot \mathscr{L}_{X} s\right\rangle \\
& \left.=2 g\left(\kappa\left(s, \mathscr{L}_{X} s\right)\right), Y\right),
\end{aligned}
$$

hence the result, since $Y$ is arbitrary.

We also "geometrise" the $\gamma$ component of a Spencer cocycle as follows. The map $\gamma: \odot{ }^{2} S \rightarrow \mathfrak{s o}(T M)$ is defined via the first cocycle condition (3.7):

$$
\gamma(s, s) X=-2 \kappa\left(s, \beta_{X} s\right)
$$

for all $s \in \Gamma(\mathbb{S})$ and $X \in \mathfrak{X}(M)$. In components, this is given by (3.40).

\subsection{Properties of Killing spinors}

From now on, we work with a background $(M, g, C, F)$. We are now almost ready to define the Killing superalgebra of such a background. However, we will see that in order for its bracket to close, the Dirac current of a Killing spinor must be a Killing vector which preserves the background fields $C$ and $F$. The following proposition will help to show this.

Proposition 19. Let $s \in \Gamma(\mathbb{S})$ be a Killing spinor field, and for notational convenience fix $\mu, \kappa, \omega$ to be the Dirac bilinears of $s$. Then the following identities hold:

$$
\begin{aligned}
& \nabla_{\mu} \mu=2 C_{\mu \alpha} \kappa^{\alpha}+\frac{1}{2} F_{A B}^{\alpha} \omega_{\mu \alpha}^{A B} \\
& \nabla_{\mu} \kappa_{\nu}=\gamma_{\mu \nu}(s, s)=2 C_{\mu \nu} \mu+\frac{1}{2} \epsilon_{\mu \nu \alpha \beta \gamma} C^{\alpha \beta} \kappa^{\gamma}+\frac{1}{4} \epsilon_{\mu \nu \alpha \beta \gamma} F_{A B}^{\alpha} \omega^{\beta \gamma A B} \\
& \nabla_{\mu} \omega_{\nu \rho}^{A B}=\epsilon_{\alpha \beta \gamma \mu[\nu} C^{\alpha \beta} \omega_{\rho]}^{\gamma}{ }_{\rho B}^{A B}+\epsilon_{\alpha \beta \gamma \nu \rho} C^{\alpha}{ }_{\mu} \omega^{\beta \gamma A B}-\frac{1}{4} \epsilon_{\mu \nu \rho \alpha \beta} F^{\alpha A B} \kappa^{\beta}+\frac{1}{2} \eta_{\mu[\nu} F_{\rho]} A B \mu \\
& +F_{[\nu}{ }_{C}^{(A} \omega_{\rho] \mu}{ }^{B) C}-F_{\mu}\left({ }_{C} \omega_{\nu \rho}^{B) C}-g_{\mu[\nu} F_{C}^{\alpha(A} \omega_{\rho] \alpha}^{B) C},\right.
\end{aligned}
$$

and in particular

$$
\begin{aligned}
\nabla^{\nu} \omega_{\nu \rho}^{A B} & =\frac{1}{2} \epsilon_{\rho \alpha \beta \gamma \delta} C^{\alpha \beta} \omega^{\gamma \delta A B}+F_{\rho}^{A B} \mu-\frac{1}{2} F_{C}^{\nu(A} \omega_{\rho \nu}{ }^{B) C} \\
\nabla_{[\mu} \omega_{\nu \rho]}^{A B} & =-\frac{1}{4} \epsilon_{\mu \nu \rho \alpha \beta} F^{\alpha A B} \kappa^{\beta} .
\end{aligned}
$$


Proof. Each of these identities follow from the Killing spinor equation. We have

$$
\nabla_{\mu}\left(\bar{s}^{A} s^{B}\right)=\bar{\nabla}_{\mu}{ }^{A} s^{B}+\bar{s}^{A} \nabla_{\mu} s^{B}=-\bar{s}^{B} \nabla_{\mu} s^{A}+\bar{s}^{A} \nabla_{\mu} s^{B}=2 \bar{s}^{[A} \nabla_{\mu} s^{B]}=2 \bar{s}^{[A}\left(\beta_{\mu} s\right)^{B]},
$$

which is equivalent to

$$
\nabla_{\mu} \mu=2 \epsilon_{A B} 2 \bar{s}^{A}\left(\beta_{\mu} s\right)^{B},
$$

and substituting in equation (4.2) for $\beta$ yields equation (5.16). Similarly, we have

$$
\nabla_{\mu} \kappa_{\nu}=2 \epsilon_{A B} \bar{s}^{A} \Gamma_{\nu}\left(\beta_{\mu} s\right)^{B}
$$

which, using cocycle condition (3.7) gives equation (5.17), and

$$
\nabla_{\mu} \omega_{\nu \rho}^{A B}=2 \bar{s}^{A} \Gamma_{\nu \rho}\left(\beta_{\mu} s\right)^{B},
$$

which after expanding and evaluating the resulting products of $\Gamma$-matrices yields equation (5.18). The expression $\nabla \omega^{A B}$ has three components - the trace, skew-symmetrisation and the elbow - which we will treat separately. The trace part gives the divergence of $\omega^{A B}$, while the skew-symmetrisation gives its exterior derivative

$$
\nabla_{[\mu} \omega_{\nu \rho]}^{A B}=\epsilon_{\alpha \beta \gamma[\mu \nu} C^{\alpha \beta} \omega_{\rho]}^{\gamma}{ }^{A B}+\epsilon_{\alpha \beta \gamma[\mu \nu} C_{\rho]}^{\alpha} \omega^{\beta \gamma A B}-\frac{1}{4} \epsilon_{\mu \nu \rho \alpha \beta} F^{\alpha A B} \kappa^{\beta},
$$

of which the first two terms cancel identically by equation (A.2), giving equation (5.20).

We highlight one of these identities in particular.

Corollary 20. If $s$ is a Killing spinor field, the gradient of its Dirac current is given by

$$
\nabla \kappa=-\gamma(s, s) \text {. }
$$

In particular, $\kappa$ is a Killing vector.

Note that this is a direct consequence of the cocycle condition equation (3.7).

\subsection{Existence of Killing superalgebras}

We can now construct the supersymmetry algebra of a supersymmetric background $(M, g, C, F)$. For such a background, we denote the space of (symplectic Majorana) Killing spinors by $\mathfrak{K}_{\overline{1}}$; that is,

$$
\mathfrak{K}_{\overline{1}}=\{s \in \Gamma(\mathbb{S}) \mid \mathscr{D} s=0\},
$$

and we also define

$$
\mathfrak{K}_{\overline{0}}=\left\{X \in \mathfrak{X}(M) \mid \mathscr{L}_{X} g=\mathscr{L}_{X} C=\mathscr{L}_{X} F=0\right\},
$$

the space of Killing vector fields which preserve $C$ and $F$. We define the bracket $[\cdot, \cdot]$ on $\mathfrak{K}=\mathfrak{K}_{\overline{0}} \oplus \mathfrak{K}_{\overline{1}}$ by extension of the following:

- the usual Lie bracket of vector fields on $\mathfrak{K}_{\overline{0}} \otimes \mathfrak{K}_{\overline{0}},[X, Y]=\mathscr{L}_{X} Y$, 
- the Dirac current on $\mathfrak{K}_{\overline{1}} \otimes \mathfrak{K}_{\overline{1}},[s, s]=\kappa_{s}$,

- the spinorial Lie derivative on $\mathfrak{K}_{\overline{0}} \otimes \mathfrak{K}_{\overline{1}},[X, s]=\mathscr{L}_{X} s$.

Theorem 21. If $(M, g, C, F)$ is a background such that $\Gamma^{\nu} R_{\mu \nu}^{\mathscr{D}}=0$, then $(\mathfrak{K},[\cdot, \cdot])$ as described above is a Lie superalgebra.

Proof. Throughout, let $s$ be a Killing spinor field and let $\mu, \kappa$ and $\omega^{A B}$ be the bilinears associated to $s$. First, note that the super skew-symmetry of the bracket follows directly from the definition. We will next show that $\mathfrak{K}$ is closed under the bracket operation. First, we have $\mathscr{L}_{\kappa} g=0$ by Corollary 20. Using the Cartan formula for the Lie derivative, we have

$$
\mathscr{L}_{\kappa} C=d \iota_{\kappa} C+\iota_{\kappa} d C
$$

but by equation (5.16), $\iota_{\kappa} C=-\frac{1}{2} d \mu-\frac{1}{4} \iota F_{A B} \omega^{A B}$, so

$$
\mathscr{L}_{\kappa} C=-\frac{1}{4} d \iota_{F_{A B}} \omega^{A B}+\iota_{\kappa} d C .
$$

In components, the first term is proportional to

$$
\begin{aligned}
\nabla_{[\mu}\left(\omega_{\nu] \rho}{ }^{A B} F_{A B}^{\rho}\right) & =\left(\nabla_{[\mu} \omega_{\nu] \rho}^{A B}\right) F_{A B}^{\rho}+\omega_{[\nu|\rho|}{ }^{A B}\left(\nabla_{\mu]} F_{A B}^{\rho}\right) \\
& =\frac{3}{2}\left(\nabla_{[\mu} \omega_{\nu \rho]}^{A B}\right) F_{A B}^{\rho}-\frac{1}{2}\left(\nabla_{\rho} \omega_{\mu \nu}{ }^{A B}\right) F_{A B}^{\rho}+\omega_{[\nu|\rho|} A B\left(\nabla_{\mu]} F_{A B}^{\rho}\right) .
\end{aligned}
$$

Using equation (5.20), the first term in this expansion vanishes identically:

$$
\frac{3}{2}\left(\nabla_{[\mu} \omega_{\nu \rho]}^{A B}\right) F_{A B}^{\rho}=-\frac{3}{8} \epsilon_{\mu \nu \rho \alpha \beta}\left(F^{\rho} \cdot F^{\alpha}\right) \kappa^{\beta}=0,
$$

because $F^{\rho} \cdot F^{\alpha}$ is symmetric. Substituting equation (5.18) into the second term of equation (5.31), three terms vanish by symmetry, leaving

$$
-\frac{1}{2}\left(\nabla_{\rho} \omega_{\mu \nu}^{A B}\right) F_{A B}^{\rho}=-\frac{1}{2}\left[\epsilon_{\alpha \beta \gamma \rho[\mu} C^{\alpha \beta} F_{A B}^{\gamma} \omega_{\nu]}^{\rho A B}+\epsilon_{\mu \nu \alpha \beta \gamma} C_{\rho}^{\alpha} F_{A B}^{\rho} \omega^{\beta \gamma A B}+\left[F^{\rho}, F_{[\mu}\right]_{B C} \omega_{\nu] \rho}^{B C}\right] .
$$

Thus

$$
\begin{aligned}
\nabla_{[\mu}\left(\omega_{\nu] \rho}^{A B} F_{A B}^{\rho}\right)= & \left(\nabla_{[\mu} F_{A B}^{\rho}-\frac{1}{2} \epsilon_{\alpha \beta \gamma \rho[\mu} C^{\alpha \beta} F_{A B}^{\gamma}\right) \omega_{\nu] \rho}^{A B} \\
& -\frac{1}{2} \epsilon_{\mu \nu \alpha \beta \gamma} C_{\rho}^{\alpha} F_{A B}^{\rho} \omega^{\beta \gamma A B}-\frac{1}{2}\left[F^{\rho}, F_{[\mu}\right]_{B C} \omega_{\nu] \rho}^{B C} .
\end{aligned}
$$

Thus $\mathscr{L}_{\kappa} C$ vanishes when $\Gamma^{\nu} R^{\mathscr{D}}{ }_{\mu \nu}=0$ by Theorem 13 . Since each $F_{A B}$ is a one-form, we can also use the Cartan formula for its Lie derivative:

$$
\mathscr{L}_{\kappa} F_{A B}=d \iota_{\kappa} F_{A B}+\iota_{\kappa} d F_{A B}
$$

which in components gives

$$
\left(\mathscr{L}_{\kappa} F_{A B}\right)_{\mu}=\nabla_{\mu}\left(\kappa^{\nu} F_{\nu A B}\right)+\kappa^{\nu} \nabla_{\nu} F_{\mu A B}-\kappa^{\nu} \nabla_{\mu} F_{\nu A B}=\left(\nabla_{\mu} \kappa^{\nu}\right) F_{\nu A B}+\kappa^{\nu} \nabla_{\nu} F_{\mu A B}
$$


Using equation (5.17), the first term on the right hand side is

$$
\begin{aligned}
\gamma_{\mu \nu} F_{A B}^{\nu} & =2 C_{\mu \nu} F_{A B}^{\nu} \mu+\frac{1}{2} \epsilon_{\mu \nu \alpha \beta \gamma} C^{\alpha \beta} F_{A B}^{\nu} \kappa^{\gamma}+\frac{1}{4} \epsilon_{\mu \nu \alpha \beta \gamma} F_{A B}^{\nu} F_{C D}^{\alpha} \omega^{\beta \gamma C D} \\
& =2 C_{\mu \nu} F_{A B}^{\nu} \mu+\frac{1}{2} \epsilon_{\mu \alpha \beta \gamma \delta} C^{\alpha \beta} F_{A B}^{\gamma} \kappa^{\delta}+\frac{1}{8} \epsilon_{\mu \alpha \beta \sigma \tau}\left[F^{\alpha}, F^{\beta}\right]_{(A}^{C} \omega_{B) C}^{\sigma \tau},
\end{aligned}
$$

where the second equality arises as follows: we have

$$
\begin{aligned}
F_{A(B}^{[\alpha} F_{C) D}^{\beta]} & =F_{[A \mid(B}^{[\alpha} F_{C) \mid D]}^{\beta]}=\frac{1}{2} \epsilon_{A D} \epsilon^{E F} F_{E(B}^{[\alpha} F_{C) F}^{\beta]}=-\frac{1}{4} \epsilon_{A D}\left[F^{\alpha}, F^{\beta}\right]_{B C}, \\
F_{A[B}^{[\alpha} F_{C] D}^{\beta]} & =\frac{1}{2} \epsilon_{B C} \epsilon^{E F} F_{A E}^{[\alpha} F_{F D}^{\beta]}=-\frac{1}{4} \epsilon_{B C}\left[F^{\alpha}, F^{\beta}\right]_{A D}
\end{aligned}
$$

and so

$$
F_{A B}^{[\alpha} F_{C D}^{\beta]}=-\frac{1}{4}\left(\epsilon_{A D}\left[F^{\alpha}, F^{\beta}\right]_{B C}+\epsilon_{B C}\left[F^{\alpha}, F^{\beta}\right]_{A D}\right),
$$

and finally symmetrising in $A B$ gives $F_{A B}^{[\alpha} F_{C D}^{\beta]}=\frac{1}{2} \epsilon_{(C \mid(A}\left[F^{\alpha}, F^{\beta}\right]_{B) \mid D)}{ }^{6}$ We thus have

$$
\begin{aligned}
\left(\mathscr{L}_{\kappa} F_{A B}\right)_{\mu}= & 2 C_{\mu \nu} F_{A B}^{\nu} \mu+\left(\nabla_{\delta} F_{\mu A B}+\frac{1}{2} \epsilon_{\mu \alpha \beta \gamma \delta} C^{\alpha \beta} F_{A B}^{\gamma}\right) \kappa^{\delta} \\
& +\frac{1}{4} \epsilon_{\mu \alpha \beta \sigma \tau}\left[F^{\alpha}, F^{\beta}\right]_{(A}^{C} \omega_{B) C}^{\sigma \tau} .
\end{aligned}
$$

By Theorem 13, this vanishes if $\Gamma^{\nu} R^{\mathscr{D}}{ }_{\mu \nu}=0$. We have thus shown that $\left[\mathfrak{K}_{\overline{1}}, \mathfrak{K}_{\overline{1}}\right] \subseteq \mathfrak{K}_{\overline{0}}$. One can easily verify that $\left[\mathfrak{K}_{\overline{0}}, \mathfrak{K}_{\overline{0}}\right] \subseteq \mathfrak{K}_{\overline{0}}$. We must show that $\left[\mathfrak{K}_{\overline{1}}, \mathfrak{K}_{\overline{0}}\right]+\left[\mathfrak{K}_{\overline{0}}, \mathfrak{K}_{\overline{1}}\right] \subseteq \mathfrak{K}_{\overline{1}}$; that is, $\mathscr{L}_{K} s \in \mathfrak{K}_{\overline{1}}$ for all $K \in \mathfrak{K}_{\overline{0}}$ and $s \in \mathfrak{K}_{\overline{1}}$. By Lemma 17 , for all $X \in \mathfrak{X}(M)$ we have

$$
\mathscr{D}_{X} \mathscr{L}_{K} s=\mathscr{D}_{[X, K]} s+\mathscr{L}_{K} \mathscr{D}_{X} s+\left(\mathscr{L}_{K} \beta\right)_{X} s=\left(\mathscr{L}_{K} \beta\right)_{X} s
$$

where we have used $\mathscr{D} s=0$, but using the expression (4.1) for $\beta$ and the Leibniz rule for the Lie derivative with respect to Clifford multiplication, we see that $\mathscr{L}_{K} \beta=0$ for all $K \in \mathfrak{K}_{\overline{0}}$. Thus $\mathscr{L}_{K} s \in \mathfrak{K}_{\overline{1}}$.

It remains to be shown only that the Jacobi identity is satisfied. For three Killing vector fields this is clear because the bracket is simply the commutator. For two Killing vector fields $X, Y$ and a Killing spinor field $s$,

$$
[s,[X, Y]]+[X,[Y, s]]-[Y,[s, X]]=-\mathscr{L}_{\mathscr{L}_{X} Y} s+\mathscr{L}_{X} \mathscr{L}_{Y} s-\mathscr{L}_{Y} \mathscr{L}_{X} s
$$

so the Jacobi identity follow from $\mathscr{L}$ being a Lie algebra representation of vector fields on spinor fields, which follows from Proposition 16. By symmetry, for the case of one Killing vector field and two Killing spinor fields, we need only consider the identity where both spinor fields are the same:

$$
[X,[s, s]]+[s,[s, X]]-[s,[X, s]]=[X, \kappa(s, s)]-\kappa\left(s, \mathscr{L}_{X} s\right)-\kappa\left(s, \mathscr{L}_{X} s\right),
$$

\footnotetext{
${ }^{6}$ This reflects the Hodge isomorphism $\wedge^{2} \odot \odot^{2} \Delta \cong \odot^{2} \Delta$ with respect to the inner product on $\odot^{2} \Delta$ induced by the symplectic product on $\Delta$.
} 
which vanishes by Lemma 18. Finally, for three Killing spinor fields, again we need only consider the case where they are all the same; using Corollary 20 this reduces to the vanishing of

$$
[[s, s], s]=\mathscr{L}_{\kappa} s=\nabla_{\kappa} s-(\nabla \kappa) s=\beta_{\kappa} s+\gamma(s, s) s,
$$

which is simply the cocycle condition (3.8).

We can now finally state the definition of the Killing superalgebra.

Definition 22. The Killing superalgebra of a background $(M, g, C, F)$ with $\Gamma^{\nu} R^{\mathscr{D}}{ }_{\mu \nu}=0$ is the Lie superalgebra $\mathfrak{K}=\mathfrak{K}_{\overline{0}} \oplus \mathfrak{K}_{\overline{1}}$ where

$$
\mathfrak{K}_{\overline{0}}=\left\{X \in \mathfrak{X}(M) \mid \mathscr{L}_{X} g=\mathscr{L}_{X} C=\mathscr{L}_{X} F=0\right\}
$$

is the space of Killing vector fields which preserve $C$ and $F$,

$$
\mathfrak{K}_{\overline{1}}=\{s \in \Gamma(\mathbb{S}) \mid \mathscr{D} s=0\}
$$

is the space of Killing spinor fields, and the bracket $[\cdot, \cdot]$ is defined above.

In the proof of Theorem 21, the vanishing Clifford trace of the curvature is used to show that the algebra closes. One might ask whether this is necessary; that is, do Killing superalgebras only exist for backgrounds which satisfy the equations of motion? Recalling Theorem 13, note that equations (4.36) and (4.39) were not necessary to close the algebra. Thus, if $F=0, d C=0$ is sufficient to close the algebra. This is the case in 5-dimensional supergravity: $C$ is (proportional to) the field strength of a Maxwell field and hence closed, so there may exist "off-shell" supersymmetric bosonic backgrounds. On the other hand, if $C=0, \nabla F=[F, F]=0$ suffices.

\section{Maximally supersymmetric backgrounds}

Throughout we work in a maximally supersymmetric background $(M, g, C, F)$ which we assume to be connected. We seek only to classify the geometries up to local isometry, so we also assume that $M$ is simply connected. Under this assumption, maximal supersymmetry is equivalent to the vanishing of the curvature $R^{\mathscr{D}}$ of the superconnection, so Theorem 14 gives necessary and sufficient conditions for maximal supersymmetry. We now work with local coordinate frames rather than local orthonormal frames, still using Greek indices.

\subsection{Maximally supersymmetric supergravity backgrounds}

Taking $F=0$ reduces the problem to the determination of maximally supersymmetric geometries in minimal 5-dimensional supergravity. These are already known in the literature. They were constructed directly in [15] and via quotients from the maximally supersymmetric backgrounds of $(1,0)$ six-dimensional supergravity in [16]. They are given by

- the near-horizon geometry of the BMPV black hole [19], 
- $A d S_{3} \times S^{2}$ and $A d S_{2} \times S^{3}$, which also arise as limits of the BMPV near-horizon solution,

- a particular Cahen-Wallach pp-wave [20],

- a 5-dimensional analogue of the Gödel universe.

In addition, in [15] there are three further candidate geometries which, as the authors already speculate, are in fact locally isometric to cases already listed above. This is easy to check by calculating their Killing superalgebras and showing that they are isomorphic to the ones above and observing, as was shown in [5] in the context of eleven-dimensional supergravity but holds more generally, that for a $\left(>\frac{1}{2}\right)$-BPS background, the Killing superalgebra, which is transitive, determines the geometry up to local isometry.

\subsection{Maximally supersymmetric backgrounds with $F \neq 0$}

By Theorem 14, the geometry is given by a nonzero parallel one-form $\varphi$ (which we now regard dually as a vector field) and the Riemann curvature is constrained by equation (4.64), which in a coordinate frame reads

$$
R_{\mu \nu \sigma \tau}=\varphi^{2} g_{\mu[\sigma} g_{\tau] \nu}-\varphi_{\mu} \varphi_{[\sigma} g_{\tau] \nu}+\varphi_{\nu} \varphi_{[\sigma} g_{\tau] \mu} .
$$

The Ricci and scalar curvatures are then

$$
R_{\mu \nu}=\frac{3}{2}\left(\varphi_{\mu} \varphi_{\nu}-\varphi^{2} g_{\mu \nu}\right) \quad \text { and } \quad R=-6 \varphi^{2},
$$

and the Weyl tensor vanishes, so the metric is conformally flat. It is also locally symmetric (hence symmetric, since $M$ is simply connected): $\nabla_{\lambda} R_{\mu \nu \sigma \tau}=0$ since $\nabla \varphi=0$. Another consequence of $\varphi \neq 0$ being parallel is that it is nowhere vanishing and $\varphi^{2}$ is constant in particular, the scalar curvature is constant. The geometry is thus determined by the causal type of $\varphi$.

\subsubsection{Spacelike $\varphi$}

When $\varphi^{2}<0, \varphi$ defines a distribution of rank 1 in $T M$ which is preserved by the holonomy of $\nabla$ since $\varphi$ is parallel. The rank-4 perpendicular distribution is also preserved by holonomy, and the metric is nondegenerate on either distribution. The de Rham-Wu decomposition theorem then allows us to decompose $(M, g)$ as a product $\left(N, g_{N}\right) \times\left(\mathbb{R},-\mathrm{d} x^{2}\right)$, where $\left(N, g_{N}\right)$ is a 4 -dimensional lorentzian manifold and $x$ is the standard coordinate on $\mathbb{R}$. We then define the symmetric tensor

$$
h_{\mu \nu}=g_{\mu \nu}-\frac{\varphi_{\mu} \varphi_{\nu}}{\varphi^{2}}
$$

so we can write the curvature tensors as

$$
\begin{aligned}
R_{\mu \nu \sigma \tau} & =\varphi^{2} h_{\mu[\sigma} h_{\tau] \nu} \\
R_{\mu \nu} & =-\frac{3}{2} \varphi^{2} h_{\mu \nu} \\
R & =-6 \varphi^{2} .
\end{aligned}
$$


The pullback of $h$ to $N$ coincides with $g_{N}$. Since $\left(N, g_{N}\right)$ is a lorentzian symmetric space with constant positive scalar curvature, ${ }^{7}$ it must be $A d S_{4}$ by a result of Cahen and Wallach [21, 22].

\subsubsection{Timelike $\varphi$}

We can treat this similarly to the spacelike case. Here, we get a decomposition $\left(\mathbb{R}, \mathrm{d} t^{2}\right) \times$ $\left(N,-g_{N}\right)$ where $t$ is the standard coordinate on $R$ and $\left(N, g_{N}\right)$ is a riemannian manifold. Note that the pullback of $h$ coincides with $-g_{N}$. The sectional curvature of $\left(N, g_{N}\right)$ is a positive constant, so it is $S^{4}$.

\subsubsection{Null $\varphi$}

In this case, the de Rham-Wu theorem cannot be used since the distribution defined by $\varphi$ is degenerate. The geometry here is a Brinkmann pp-wave space - a lorentzian manifold with a parallel null vector field. The curvature tensors reduce to

$$
\begin{aligned}
R_{\mu \nu \sigma \tau} & =-g_{\mu[\sigma} \varphi_{|\nu|} \varphi_{\tau]}+g_{\nu[\sigma} \varphi_{|\mu|} \varphi_{\tau]} \\
R_{\mu \nu} & =\frac{3}{2} \varphi_{\mu} \varphi_{\nu}
\end{aligned}
$$

and $R=0$. Thus $(M, g)$ is a scalar-flat lorentzian symmetric space; by the Cahen-Wallach theorem $[21,22]$ it is a Cahen-Wallach pp-wave $C W_{5}(A)$. Such a space has a coordinate system $\left(x^{+}, x^{-}, x^{1}, x^{2}, x^{3}\right)$ in which the metric is given by

$$
g=2 \mathrm{~d} x^{+} \mathrm{d} x^{-}-\left(\sum_{i, j=1}^{3} A_{i j} x^{i} x^{j}\right)\left(\mathrm{d} x^{-}\right)^{2}-\sum_{i=1}^{3}\left(\mathrm{~d} x^{i}\right)^{2},
$$

where $A \in \odot^{2} \mathbb{R}^{3}$. We take $\varphi=\partial_{+}$to be the distinguished parallel null vector field. This metric is scalar-flat for any $A$, and the non-vanishing components of the Riemann and Ricci tensors are $R_{i-j-}=-A_{i j}$ and $R_{--}=-\sum_{i=1}^{3} A_{i i}$. The non-vanishing components of the Weyl tensor are given by the trace-free part of $A$ : namely,

$$
W_{i-j-}=-A_{i j}+\frac{1}{3} \delta_{i j} \sum_{k=1}^{3} A_{k k} .
$$

We already saw that the Weyl tensor for a maximally supersymmetric geometry vanishes, so we have $A_{i j}=a \delta_{i j}$ where $a=\frac{1}{3} \sum_{k=1}^{3} A_{k k}$. Now, comparing with equations (6.5) for $\varphi=\partial_{+}$, we find that $a=-\frac{1}{2}$. We have thus shown the following.

Theorem 23. Let $(M, g, C, F)$ be a maximally supersymmetric 5-dimensional background. If $F=0$ then $(M, g)$ is a maximally supersymmetric background of minimal 5-dimensional supergravity. If $F \neq 0$ then $C=0$ and $F=\varphi \otimes r$ for some one-form $\varphi$ and some $r \in \mathfrak{s p}(1)$, and up to local isometry,

- If $\varphi^{2}>0,(M, g)=\mathbb{R}^{1,0} \times S^{4}$, where $S^{4}$ is the round sphere with scalar curvature $6 \varphi^{2}$ (radius $\sqrt{\frac{2}{\varphi^{2}}}$ ),

\footnotetext{
${ }^{7}$ One might object that $A d S_{4}$ has negative scalar curvature; however, this is only true in mostly-positive signature. The Ricci tensor is invariant under the homothety $g \rightarrow-g$, while the scalar curvature undergoes a change of sign, thus $A d S_{4}$ has positive curvature in our conventions.
} 
- If $\varphi^{2}<0,(M, g)=A d S_{4} \times \mathbb{R}^{0,1}$, where $A d S_{4}$ is the 4-dimensional anti-de Sitter spacetime with scalar curvature $6 \varphi^{2}$ (cosmological constant $\Lambda=\frac{3}{2} \varphi^{2}$ ),

- If $\varphi^{2}=0,(M, g)$ is a Cahen-Wallach pp-wave with coordinates $\left(x^{+}, x^{-}, x^{1}, x^{2}, x^{3}\right)$ in which $\varphi=\partial_{+}$and

$$
g=2 \mathrm{~d} x^{+} \mathrm{d} x^{-}+\frac{1}{2}\left(\sum_{i=1}^{3}\left(x^{i}\right)^{2}\right)\left(\mathrm{d} x^{-}\right)^{2}-\sum_{i=1}^{3}\left(\mathrm{~d} x^{i}\right)^{2} .
$$

We note that this result here is (up to a rescaling of $\varphi$ and $r$ ) precisely the 5-dimensional analogue of Theorem 27 part (ii) in [14].

\subsection{Killing superalgebras}

In this section we will explicitly describe the Killing superalgebras of the maximally supersymmetric backgrounds as filtered subdeformations of the five-dimensional minimal Poincaré superalgebra. As explained in [5] in the context of eleven-dimensional supergravity, the Killing superalgebra is generated by sections of a supervector bundle $\mathscr{E}=\mathscr{E}_{0} \oplus \mathscr{E}_{\overline{1}}$, where

$$
\mathscr{E}_{\overline{0}}=T M \oplus \mathfrak{s o}(T M) \quad \text { and } \quad \mathscr{E}_{\overline{1}}=\mathbb{S}
$$

which are parallel relative to a superconnection $\mathscr{D}$ which agrees on sections of $\mathscr{E}_{\overline{0}}$ with the Killing transport connection $[23,24]$ and on sections of $\mathscr{E}_{1}$ with the connection given by equation (4.3). There might be in addition additional (tensorial) constraints on the sections of $\mathscr{E}$.

For the case at hand, the Killing superalgebra is a filtered Lie superalgebra whose underlying vector space is $V \oplus S \oplus \mathfrak{h}$, where $\mathfrak{h} \subset \mathfrak{s o}(V)$ is a subalgebra. The Lie brackets of the Killing superalgebra are defined by

$$
\begin{aligned}
{[A, B] } & =A B-B A \\
{[A, s] } & =\frac{1}{2} \omega_{A} \cdot s \\
{[A, v] } & =A v+\underbrace{\left[A, \lambda_{v}\right]-\lambda_{A v}}_{\in \mathfrak{h}}
\end{aligned}
$$

$$
\begin{aligned}
{[s, s] } & =\kappa(s)+\underbrace{\gamma^{\Phi}(s, s)-\lambda_{\kappa(s, s)}}_{\in \mathfrak{h}} \\
{[v, s] } & =\beta_{v}^{\Phi} s+\frac{1}{2} \omega_{\lambda_{v}} \cdot s \\
{[v, w] } & =\underbrace{\lambda_{v} w-\lambda_{w} v}_{\in V}+\underbrace{R(v, w)+\left[\lambda_{v}, \lambda_{w}\right]-\lambda_{\lambda_{v} w-\lambda_{w} v}}_{\in \mathfrak{h}},
\end{aligned}
$$

for all $A, B \in \mathfrak{h}, s \in S$ and $v, w \in V$. Here $\beta^{\Phi}+\gamma^{\Phi}$ is the normalised cocycle (with $\alpha=0$ ) where $\Phi$ stands for the generic additional fields (here $C$ and $F$ ) and $\mathfrak{h}=\mathfrak{s o}(V) \cap \mathfrak{s t a b}(\Phi)$ is the Lie algebra of the stabiliser of $\Phi$ in $\mathrm{SO}(V)$. In addition, $R$ is the Riemann curvature tensor, induced from a map $\wedge^{2} V \rightarrow \mathfrak{s o}(V)$. Finally, $\lambda: V \rightarrow \mathfrak{s o}(V)$ is defined only up to a linear map $V \rightarrow \mathfrak{h}$, so it is to be thought of more precisely as a linear map $V \rightarrow \mathfrak{s o}(V) / \mathfrak{h}$. 


\subsubsection{Killing superalgebras for maximally supersymmetric supergravity back- grounds}

For these backgrounds, the normalised cocycle is given by equation (3.40) with $F=0$. In components, we have

$$
\begin{aligned}
\beta_{\mu}^{\Phi} & =\frac{1}{8} C^{\sigma \tau}\left(\Gamma_{\mu} \Gamma_{\sigma \tau}-3 \Gamma_{\sigma \tau} \Gamma_{\mu}\right) \\
\gamma_{\mu \nu}^{\Phi} & =2 \mu C_{\mu \nu}+\frac{1}{2} \epsilon_{\mu \nu \rho \sigma \tau} \kappa^{\rho} C^{\sigma \tau} .
\end{aligned}
$$

Letting $\gamma(s, s):=\gamma^{\Phi}(s, s)-\lambda_{\kappa(s, s)}$, we find that

$$
\gamma_{\mu \nu}=2 \mu C_{\mu \nu}+\frac{1}{2} \epsilon_{\mu \nu \rho \sigma \tau} \kappa^{\rho} C^{\sigma \tau}-\kappa^{\rho} \lambda_{\rho \mu \nu}
$$

where $\lambda_{\rho \mu \nu}=-\lambda_{\rho \nu \mu}$. Demanding that $\gamma(s, s) \in \mathfrak{h}$ is tantamount to demanding the vanishing of the Clifford commutator

$$
\left[\gamma_{\mu \nu} \Gamma^{\mu \nu}, C_{\sigma \tau} \Gamma^{\sigma \tau}\right]=0,
$$

since that is, up to inconsequential factors, the action of $\gamma(s, s)$ on $C$. It is clear that if we define $\lambda$ by

$$
\lambda_{\rho \mu \nu}=\frac{1}{2} \epsilon_{\rho \mu \nu \sigma \tau} C^{\sigma \tau},
$$

then $\gamma(s, s) \in \mathfrak{h}$, where $\gamma_{\mu \nu}=2 \mu C_{\mu \nu}$. We remark that $\lambda$ is $\mathfrak{h}$-equivariant, so that the $\mathfrak{h}$ component of the $[A, v]$ bracket in equation (6.10) is absent.

Defining $\beta_{v}:=\beta_{v}^{\Phi}+\frac{1}{2} \omega_{\lambda_{v}}$, we see that in components

$$
\begin{aligned}
\beta_{\mu} & =\beta_{\mu}^{\Phi}+\frac{1}{4} \lambda_{\mu \sigma \tau} \Gamma^{\sigma \tau} \\
& =\frac{1}{8} C^{\alpha \beta}\left(\Gamma_{\mu} \Gamma_{\alpha \beta}-3 \Gamma_{\alpha \beta} \Gamma_{\mu}+\epsilon_{\mu \sigma \tau \alpha \beta} \Gamma^{\sigma \tau}\right) \\
& =\frac{1}{4} C^{\alpha \beta} \epsilon_{\mu \alpha \beta \sigma \tau} \Gamma^{\sigma \tau}+C_{\mu \nu} \Gamma^{\nu} .
\end{aligned}
$$

Now $\alpha(v, w):=\lambda_{v} w-\lambda_{w} v$ is given in components by

$$
\alpha_{\mu \nu \rho}=-\epsilon_{\mu \nu \rho \sigma \tau} C^{\sigma \tau} .
$$

Defining $\rho: \wedge^{2} V \rightarrow \mathfrak{s o}(V)$ by $\rho(v, w):=R(v, w)+\left[\lambda_{v}, \lambda_{w}\right]-\lambda_{\alpha(v, w)}$, we can write the Lie brackets of the Killing superalgebra as follows:

$$
\begin{aligned}
{[A, B] } & =A B-B A & {[s, s] } & =\kappa(s)+\gamma(s, s) \\
{[A, s] } & =\frac{1}{2} \omega_{A} \cdot s & {[v, s] } & =\beta_{v} s \\
{[A, v] } & =A v & {[v, w] } & =\alpha(v, w)+\rho(v, w),
\end{aligned}
$$

and determine $\rho$ by the Jacobi identity and check that it maps to $\mathfrak{h}$. Most of the components of the Jacobi identity are already satisfied by construction:

- $[\mathfrak{h}, \mathfrak{h},-]$ because $V \oplus S \oplus \mathfrak{h}$ is an $\mathfrak{h}$-module, 
- $[\mathfrak{h}, S, S]$ because $\gamma$ is $\mathfrak{h}$-equivariant,

- $[\mathfrak{h}, S, V]$ because $\beta$ is $\mathfrak{h}$-equivariant,

- $[S, S, S]$ because of the Spencer cocycle condition, and

- the $V$-component of $[S, S, V]$ because of the Spencer cocycle condition.

We will use the $[S, V, V]$ Jacobi to define $\rho$ and then check that $\rho$ is $\mathfrak{h}$-equivariant and maps to $\mathfrak{h}$ which means that the $[\mathfrak{h}, V, V]$ Jacobi is satisfied. Finally, we have to check that the $\mathfrak{h}$-component of the $[S, S, V]$ Jacobi as well as $[V, V, V]$ Jacobi are satisfied.

The $[S, V, V]$ Jacobi says that for all $v, w \in V$ and $s \in S$,

$$
[v,[w, s]]-[w,[v, s]] \stackrel{!}{=}[[v, w], s]
$$

which is equivalent to

$$
\frac{1}{2} \omega_{\rho(v, w)} \stackrel{!}{=}\left[\beta_{v}, \beta_{w}\right]-\beta_{\alpha(v, w)} .
$$

This requires that the r.h.s. should belong to $\wedge^{2} V \subset C \ell(V)$, which can be checked to be the case. In components, the above condition is

$$
\frac{1}{4} \rho_{\mu \nu \alpha \beta} \Gamma^{\alpha \beta} \stackrel{!}{=}\left[\beta_{\mu}, \beta_{\nu}\right]-\alpha_{\mu \nu}{ }^{\rho} \beta_{\rho}
$$

and after a short calculation (which we omit) results in

$$
\rho_{\mu \nu \alpha \beta}=4 C_{\mu \nu} C_{\alpha \beta} .
$$

It follows that $\rho$ is $\mathfrak{h}$-equivariant and moreover that it lands in $\mathfrak{h}$. Therefore the $[\mathfrak{h}, V, V]$ Jacobi is satisfied. It is straightforward, if somewhat tedious, to check that the rest of the Jacobi identity is satisfied.

In summary, the Killing superalgebra of a maximally supersymmetric background $(M, g, C)$ of minimal 5-dimensional supergravity is the filtered Lie superalgebra with underlying vector space $\mathfrak{g}=V \oplus S \oplus \mathfrak{h}$, where $\mathfrak{h}=\mathfrak{s o}(V) \cap \mathfrak{s t a b}(C)$, whose brackets are given for all $A, B \in \mathfrak{h}, s \in S$ and $v, w \in V$ by equation (6.17), with

$$
\begin{aligned}
\alpha_{\mu \nu \rho} & =-\epsilon_{\mu \nu \rho \sigma \tau} C^{\sigma \tau} \\
\beta_{\mu} & =\frac{1}{4} \epsilon_{\mu \alpha \beta \sigma \tau} C^{\alpha \beta} \Gamma^{\sigma \tau}+C_{\mu \nu} \Gamma^{\nu} \\
\gamma_{\mu \nu} & =2 \mu C_{\mu \nu} \\
\rho_{\mu \nu \alpha \beta} & =4 C_{\mu \nu} C_{\alpha \beta} .
\end{aligned}
$$

\subsubsection{Killing superalgebras for maximally supersymmetric backgrounds with $\boldsymbol{F} \neq \mathbf{0}$}

For these backgrounds, $C=0$ and $F=\varphi \otimes r$ where $\varphi$ is a parallel vector field (or one-form) and $r \in \mathfrak{s p}(1)$ is a fixed element of the R-symmetry Lie algebra. The normalised cocycle $\beta^{\Phi}+\gamma^{\Phi}$ can be read off from equation (3.40):

$$
\begin{aligned}
\beta^{\Phi}(v, s)^{A} & =-\frac{1}{8}(v \cdot \varphi+3 \varphi \cdot v) \cdot r_{B}^{A} s^{B} \\
\gamma^{\Phi}(s, s)_{\mu \nu} & =\frac{1}{4} \epsilon_{\mu \nu \rho}{ }^{\sigma \tau} \varphi^{\rho} r_{A B} \omega_{\sigma \tau}^{A B} .
\end{aligned}
$$


It follows that $\gamma(s, s) \in \mathfrak{h}=\mathfrak{s o}(V) \cap \mathfrak{s t a b}(\varphi)$ : indeed,

$$
\gamma_{\mu \nu}^{\Phi} \varphi^{\nu}=\frac{1}{4} \varphi^{\nu} \varphi^{\rho} \epsilon_{\mu \nu \rho}^{\sigma \tau} r_{A B} \omega_{\sigma \tau}^{A B}=0,
$$

by symmetry. This means that we can choose $\lambda=0$ and hence $\alpha=0, \beta=\beta^{\Phi}$ and $\gamma=\gamma^{\Phi}$ and the Lie brackets of the Killing superalgebra are given by

$$
\begin{aligned}
{[A, B] } & =A B-B A & {[s, s] } & =\kappa(s)+\gamma \\
{[A, s] } & =\frac{1}{2} \omega_{A} \cdot s & {[v, s] } & =\beta_{v} s \\
{[A, v] } & =A v & {[v, w] } & =\rho(v, w),
\end{aligned}
$$

subject to the Jacobi identity, which will determine $\rho$.

As in the case of the supergravity backgrounds, most of the components of the Jacobi identity are already satisfied. The $[\mathfrak{h}, V, V]$ component will follow from the $\mathfrak{h}$-component of the $[S, S, V]$ Jacobi. Indeed, this component says that

$$
\rho(v, \kappa(s, s)) \stackrel{!}{=} 2 \gamma(\beta(v, s), s) .
$$

If we can solve this equation for $\rho$, which basically means that the r.h.s. only depends on $\kappa(s, s)$ and not on either $\mu$ nor $\omega^{A B}$, then $\rho$ is indeed $\mathfrak{h}$-equivariant, since so are $\beta$ and $\gamma$, and $\rho$ maps to $\mathfrak{h}$, since so does $\gamma$. The other two Jacobi components which need to be satisfied are the $[S, V, V]$ component:

$$
\beta(v, \beta(w, s))-\beta(w, \beta(v, s)) \stackrel{!}{=}[\rho(v, w), s]
$$

and the $[V, V, V]$ component:

$$
\rho(u, v) w+\rho(v, w) u+\rho(w, u) v \stackrel{!}{=} 0 .
$$

We actually prefer to derive $\rho$ from the $[S, V, V]$ Jacobi and check the other two. A straightforward (if tedious) calculation shows that

$$
\rho_{\mu \nu \sigma \tau}=\varphi^{2} g_{\mu[\sigma} g_{\tau] \nu}-\varphi_{\mu} \varphi_{[\sigma} g_{\tau] \nu}+\varphi_{\nu} \varphi_{[\sigma} g_{\tau] \mu},
$$

agreeing, as expected, with the Riemann tensor (6.1). It follows that $\rho$ is $\mathfrak{h}$-equivariant and we check that it does map to $\mathfrak{h}$ : indeed, a short calculation shows that $\rho_{\mu \nu \sigma \tau} \varphi^{\tau}=0$, with terms cancelling pairwise. Similarly, one checks that $\rho_{[\mu \nu \sigma] \tau}=0$, which shows that the $[V, V, V]$ Jacobi is satisfied and, finally, that equation (6.26) is too.

In summary, the Killing superalgebra of the maximally supersymmetry backgrounds with $C=0$ is given by equation (6.25) with $\mathfrak{h}=\mathfrak{s o}(V) \cap \mathfrak{s t a b}(\varphi)$ and

$$
\begin{aligned}
\beta_{\mu}{ }^{A}{ }_{B} & =-\frac{1}{8} \varphi^{\nu}\left(\Gamma_{\mu} \Gamma_{\nu}+3 \Gamma_{\nu} \Gamma_{\mu}\right) r_{B}^{A} \\
\gamma_{\mu \nu} & =\frac{1}{4} \epsilon_{\mu \nu \rho \sigma \tau} \varphi^{\rho} \omega^{\sigma \tau}
\end{aligned}
$$

where we have introduced the shorthand $\omega_{\sigma \tau}:=r_{A B} \omega_{\sigma \tau}^{A B}$ and where $\rho$ is given by equation (6.29). 


\section{Acknowledgments}

We are very grateful to Paul de Medeiros and Andrea Santi for many useful conversations about supersymmetry and Spencer cohomology. We are grateful to an anonymous referee for suggesting ways to improve the exposition. The research of $\mathrm{AB}$ is partially supported by an STFC postgraduate studentship. Part of this work was previously submitted by AB in a dissertation as part of an MSc degree under the University of Edinburgh School of Physics and Astronomy and the Higgs Centre for Theoretical Physics, with tuition fees paid by a Highly Skilled Workforce Scholarship from the University of Edinburgh.

\section{A Tensorial identities for 2-forms}

We collect here some identities which are required for various calculations in the main body of this paper. Let $(M, g)$ be a 5-dimensional lorentzian manifold and let $A, B \in \Omega^{2}(M)$. We work in a local orthonormal frame, starting with the identity

$$
\epsilon_{\alpha \beta \gamma[\mu \nu} A_{\rho]}^{\alpha} B^{\beta \gamma}+\frac{2}{3} \epsilon_{\mu \nu \rho \alpha \beta} A^{\alpha \delta} B_{\delta}^{\beta}=0,
$$

which can be verified by contracting the left hand side with $\epsilon^{\mu \nu \rho \sigma \tau}$. It then follows that

$$
\epsilon_{\alpha \beta \gamma[\mu \nu} A_{\rho]}^{\alpha} B^{\beta \gamma}+\epsilon_{\alpha \beta \gamma[\mu \nu} A^{\alpha \beta} B_{\rho]}^{\gamma}=0 .
$$

We can then show that

$$
\epsilon_{\nu \rho \alpha \beta \gamma}\left(A_{\mu}^{\alpha} B^{\beta \gamma}+A^{\alpha \beta} B_{\mu}^{\gamma},\right)+\eta_{\mu[\nu} \epsilon_{\rho] \alpha \beta \gamma \delta} A^{\alpha \beta} B^{\gamma \delta}=0
$$

by contracting the left hand side with $\epsilon^{\nu \rho \sigma \tau \chi}$ and using equation (A.2). In particular, we have

$$
\begin{aligned}
\epsilon_{\alpha \beta \gamma[\mu \nu} A_{\rho]}^{\alpha} A^{\beta \gamma} & =0, \\
\epsilon_{\alpha \beta \gamma \nu \rho} A_{\mu}{ }^{\alpha} A^{\beta \gamma}+\frac{1}{2} \eta_{\mu[\nu} \epsilon_{\rho] \alpha \beta \gamma \delta} A^{\alpha \beta} A^{\gamma \delta} & =0 .
\end{aligned}
$$

Open Access. This article is distributed under the terms of the Creative Commons Attribution License (CC-BY 4.0), which permits any use, distribution and reproduction in any medium, provided the original author(s) and source are credited.

\section{References}

[1] V. Pestun et al., Localization techniques in quantum field theories, J. Phys. A 50 (2017) 440301 [arXiv: 1608.02952] [INSPIRE].

[2] G. Festuccia and N. Seiberg, Rigid Supersymmetric Theories in Curved Superspace, JHEP 06 (2011) 114 [arXiv:1105.0689] [INSPIRE].

[3] J.M. Figueroa-O'Farrill, P. Meessen and S. Philip, Supersymmetry and homogeneity of M-theory backgrounds, Class. Quant. Grav. 22 (2005) 207 [hep-th/0409170] [INSPIRE].

[4] J. Figueroa-O'Farrill and N. Hustler, The homogeneity theorem for supergravity backgrounds, JHEP 10 (2012) 014 [arXiv: 1208.0553] [INSPIRE]. 
[5] J. Figueroa-O'Farrill and A. Santi, On the algebraic structure of Killing superalgebras, Adv. Theor. Math. Phys. 21 (2017) 1115 [arXiv:1608.05915] [InSPIRE].

[6] J. Figueroa-O'Farrill and R. Grassie, Kinematical superspaces, JHEP 11 (2019) 008 [arXiv: 1908.11278] [INSPIRE].

[7] B. Zumino, Nonlinear Realization of Supersymmetry in de Sitter Space, Nucl. Phys. B 127 (1977) 189 [INSPIRE].

[8] M. Blau, Killing spinors and SYM on curved spaces, JHEP 11 (2000) 023 [hep-th/0005098] [INSPIRE].

[9] S.-J. Cheng and V.G. Kac, Generalized Spencer cohomology and filtered deformations of $\mathbb{Z}$-graded Lie superalgebras, Adv. Theor. Math. Phys. 2 (1998) 1141 [math/9805039] [INSPIRE].

[10] S.-J. Cheng and V.G. Kac, Addendum: Generalized Spencer cohomology and filtered deformations of $\mathbb{Z}$-graded Lie superalgebras, Adv. Theor. Math. Phys. 8 (2004) 697.

[11] J. Figueroa-O'Farrill and A. Santi, Spencer cohomology and 11-dimensional supergravity, Commun. Math. Phys. 349 (2017) 627 [arXiv:1511.08737] [INSPIRE].

[12] J. Figueroa-O'Farrill and A. Santi, Eleven-dimensional supergravity from filtered subdeformations of the Poincaré superalgebra, J. Phys. A 49 (2016) 295204 [arXiv: 1511.09264] [INSPIRE].

[13] P. de Medeiros, J. Figueroa-O'Farrill and A. Santi, Killing superalgebras for Lorentzian four-manifolds, JHEP 06 (2016) 106 [arXiv: 1605.00881] [INSPIRE].

[14] P. de Medeiros, J. Figueroa-O'Farrill and A. Santi, Killing superalgebras for Lorentzian six-manifolds, J. Geom. Phys. 132 (2018) 13 [arXiv: 1804.00319] [InSPIRE].

[15] J.P. Gauntlett, J.B. Gutowski, C.M. Hull, S. Pakis and H.S. Reall, All supersymmetric solutions of minimal supergravity in five- dimensions, Class. Quant. Grav. 20 (2003) 4587 [hep-th/0209114] [inSPIRE].

[16] A. Chamseddine, J.M. Figueroa-O'Farrill and W. Sabra, Supergravity vacua and Lorentzian Lie groups, hep-th/0306278 [INSPIRE].

[17] Y. Kosmann, Dérivées de Lie des spineurs, Ann. Mat. Pura Appl. 91 (1972) 317.

[18] J.M. Figueroa-O'Farrill, On the supersymmetries of Anti-de Sitter vacua, Class. Quant. Grav. 16 (1999) 2043 [hep-th/9902066] [INSPIRE].

[19] J.C. Breckenridge, R.C. Myers, A.W. Peet and C. Vafa, D-branes and spinning black holes, Phys. Lett. B 391 (1997) 93 [hep-th/9602065] [INSPIRE].

[20] P. Meessen, A small note on P P wave vacua in six-dimensions and five-dimensions, Phys. Rev. D 65 (2002) 087501 [hep-th/0111031] [INSPIRE].

[21] M. Cahen and N. Wallach, Lorentzian symmetric spaces, Bull. Am. Math. Soc. 76 (1970) 585.

[22] M. Cahen and M. Parker, Parallélismes absolus des variétés lorentziennes, Annales Inst. Fourier 27 (1977) 251.

[23] B. Kostant, Holonomy and the Lie algebra of infinitesimal motions of a Riemannian manifold, Trans. Am. Math. Soc. 80 (1955) 528.

[24] R.P. Geroch, Limits of spacetimes, Commun. Math. Phys. 13 (1969) 180 [inSPIRE]. 